\title{
Adaptive Threshold Generation in Robust Fault Detection using Interval Models: Time and Frequency Domain Approaches
}

\author{
Vicenç Puig, Saúl Montes de Oca and Joaquim Blesa \\ Advanced Control Systems Group, Institut de Robòtica i Informática Industrial (CSIC-UPC), Llorens i Artigas, 4-6, \\ 08028 Barcelona, Spain, e-mail: vicenc.puig@upc.edu
}

\begin{abstract}
SUMMARY
In this paper, robust fault detection is addressed based on evaluating the residual energy that it is compared against worst-case value (threshold) generated considering parametric modelling uncertainty using interval models. The evaluation of the residual/threshold energy can be done either in the time or frequency domain. This paper proposes methods to compute such energy in the two domains. The first method generates the adaptive threshold in the time domain through determining the worst-case time evolution of the residual's energy using a zonotope-based algorithm. The second method evaluates the worst-case energy evolution in the frequency domain using the Kharitonov polynomials. Results obtained using both approaches are related through the Paserval's Theorem. Finally, two application examples (a smart servoactuator and a two degrees of freedom helicopter) will be used to assess the validity of the proposed approaches and compare the results obtained.
\end{abstract}

Received ...

KEY WORDS: Robust Fault Detection, Adaptive Threshold, Zonotopes, Time-Domain, FrequencyDomain

\section{INTRODUCTION}

Model-based fault detection of dynamic processes is based on the use of models to check the consistency of observed behaviours trough installed sensors. However, when building a model of a dynamic process to monitor its behavior, there is always a mismatch between the modeled and real behavior. This is because some effects are neglected, some non-linearities are linearised in order to simplify the model, some parameters have tolerance when they are compared with several units of the same component, some errors in parameters or in the structure of the model are introduced in the model calibration process, etc. These modeling errors introduce uncertainty in the model and interfere with the fault detection. A fault detection algorithm able to handle uncertainty is called robust [11]. The robustness of fault detection algorithm is the degree of sensitivity to faults compared to the degree of sensitivity to uncertainty. These last years, research on robust fault detection methods has been very active in the fault detection and isolation (FDI) community. One of the most developed families of approaches, called active, is based on generating residuals, which 
are insensitive to uncertainty, while at the same time sensitive to faults [11]. On the other hand, there is a second family of approaches, called passive, which enhances the robustness of the fault detection system at the decision-making stage. Several techniques have been used, but most of them are based on using an adaptive threshold at the decision-making stage [21, 28, 24, 50].

Adaptive threshold generation has been a very active area of research in robust fault detection. Since the seminal works of Horak [28] in case of structured uncertainty and Emami-Naeini [21] in case of unstructured uncertainty, many researchers have analyzed how the effect of model uncertainty should be taken into account when determining the optimal threshold to be used in residual evaluation. Two approaches have been followed: the first based on determining the optimal threshold in the time domain [28, 45, 29] and the second in the frequency domain $[21,18,24,27,47]$. In the time domain approach, the uncertainty has been mainly modeled using structured uncertainty ("interval models"), while in the frequency domain approach first unstructured uncertainty was used [28] and later structured uncertainty has been also taken into account $[27,47]$. However, the connection between time and frequency domain approaches has not been established yet. In Hamelin [27], it is suggested as a future research. One of the difficulties in connecting both approaches is that in the time-domain approaches, the optimal threshold is directly determined through the interval obtained by propagating parameter uncertainty to the residual. On the other hand, in the frequency domain approaches, the optimal threshold is determined by the worst-case evaluation of the residual's energy on a time window. This difference can be explained because the optimal threshold, in frequency or time domain, is computed using the most direct form in each domain.

The aim of this paper is to introduce and compare two approaches for evaluating adaptive thresholds bounding residual energy in case of considering parametric uncertainty by means of interval models. The first approach works in the time domain while the second in the frequency domain. The time domain approach generates the adaptive threshold through determining the worstcase time evolution of the residual's energy using a zonotope-based algorithm. On the other hand, the frequency domain approach evaluates the worst-case energy evolution in the frequency domain using the Kharitonov polynomials. Results obtained using both approaches are related through the Paserval's Theorem. Finally, two application examples (a smart servoactuator and a two degrees of freedom helicopter) is used to assess the validity of the proposed approaches and compare the results obtained.

The structure of this paper is the following: In Section 2, the robust fault detection problem using energy residual evaluation by considering parametric uncertainties by means of interval models is introduced. In Section 3, an energy residual threshold generation approach using the worst-case response in time domain computed trough a zonotope based algorithm is presented. In Section 4, an energy residual threshold generation approach in the frequency domain using $H_{\infty}$ and Kharitonov polynomials is presented . Relation between time-domain and frequency-domain residual evaluation using Parseval's Theorem is presented in Section 5. Section 6 presents the relation between time and frequency domains of adaptive thresholding methods. In Section 7, an application example based on the DAMADICS servoactuator benchmark is presented and used to assess the validity of the results derived in the paper. In Section 8, a second illustrative example based on the Twin-Rotor MIMO System (TRMS) is introduced. Finally, in Section 9, the main conclusions are summarised. 


\section{PROBLEM STATEMENT}

\subsection{Problem set-up}

Let us consider that the system to be monitored can be described by a MIMO linear uncertain dynamic model expressed as follows:

$$
\begin{aligned}
\dot{x}(t) & =A(\tilde{\theta}) x(t)+B(\tilde{\theta}) u_{0}(t)+F_{a}(\tilde{\theta}) f_{a}(t) \\
y(t) & =C(\tilde{\theta}) x(t)+F_{y}(\tilde{\theta}) f_{y}(t)
\end{aligned}
$$

where $u_{0}(t) \in \mathfrak{R}^{n_{u}}$ is the real system input, $y(t) \in \mathfrak{R}^{n_{y}}$ is the system output, $x(t) \in \mathfrak{R}^{n_{x}}$ is the statespace vector, $f_{a}(t) \in \mathfrak{R}^{n_{u}}$ and $f_{y}(t) \in \mathfrak{R}^{n_{y}}$ represents faults in the system actuators and output sensors, respectively. $A(\tilde{\theta}), B(\tilde{\theta}), C(\tilde{\theta}), D(\tilde{\theta}), F_{a}(\tilde{\theta})$ and $F_{y}(\tilde{\theta})$ are matrices of appropriate dimensions where $\tilde{\theta}$ is the vector of system parameters.

The system (1)-(2) is monitored using a linear observer with Luenberger structure that uses an interval model of the system, i.e., a model with parameters bounded by intervals

$$
\underline{\theta_{i}} \leq \theta_{i} \leq \overline{\theta_{i}}, \quad i=1, \ldots, n_{\theta}
$$

that represent the uncertainty about the exact knowledge of real system parameters $\tilde{\theta}$. ${ }^{*}$ This observer, known as an interval observer, is expressed as follows [37]:

$$
\begin{aligned}
\dot{\hat{x}}(t, \theta) & =(A(\theta)-L C(\theta)) \hat{x}(t, \theta)+B(\theta) u(t)+L y(t) \\
& =A_{0}(\theta) \hat{x}(t, \theta)+B(\theta) u(t)+L y(t) \\
\hat{y}(t, \theta) & =C(\theta) \hat{x}(t, \theta)
\end{aligned}
$$

where $u(t)$ is the measured system input vector, $\dot{\hat{x}}(t, \theta)$ is the estimated system state vector, $\hat{y}(t, \theta)$ is the estimated system output vector and $A_{0}(\theta)=A(\theta)-L C(\theta)$ is the observer matrix. Notice that the measured system input, $u(t)$, includes the effect of faults in the input sensors and can be written as:

$$
u(t)=u_{0}(t)+F_{u}(\theta) f_{u}(t)
$$

where $u_{0}(t)$ is the real system input, $f_{u}(t) \in \mathfrak{R}^{n_{u}}$ is the input sensor fault while $F_{u}(\theta) \in \mathfrak{R}^{n_{u} \times n_{u}}$ is its associated matrix.

The observer gain matrix $L \in \mathfrak{R}^{n_{x} \times n_{y}}$ is designed to stabilize the matrix $A_{0}(\theta)$ and to guarantee a desired performance regarding fault detection for all $\theta \in \Theta$ [12]. The effect of the uncertain parameters $\theta$ on the observer temporal response $\hat{y}(t, \theta)$ will be bounded using an interval satisfying

$$
\hat{y}(t, \theta) \in[\underline{\hat{y}}(t), \overline{\hat{y}}(t)]
$$

\footnotetext{
*The intervals for uncertain parameters can be inferred from real data using set-membership parameter estimation algorithms [38, 43].
} 
in a non-faulty case. Such interval can be computed independently for each output $i$ ( $i=$ $\left.1, \ldots, n_{y}\right)$, neglecting couplings among outputs, as follows

$$
\underline{\hat{y}_{i}}(t)=\min _{\theta \in \Theta}\left(\hat{y}_{i}(t, \theta)\right) \quad \text { and } \quad \overline{\hat{y}}_{i}(t)=\max _{\theta \in \Theta}\left(\hat{y}_{i}(t, \theta)\right)
$$

subject to the observer equations given by (4). The optimisation problems (7) are solved using numerical methods as in [46]. However, in this paper an alternative approach based on the use of zonotopes (see Algorithm 1 in Section 3 will be used that is more efficient from the computational point of view).

R2-1, R2-2

\subsection{Observer design}

The design of the interval observer (4) can be solved with the LMI pole placement technique [12, $13,48]$, that allows to locate the poles of the observer in a subregion of the left half-plane using a LMI region assuming the pair $(A(\theta), C(\theta))$ is detectable for all $\theta \in \Theta$.

In particular, a disk LMI region called $\mathcal{D}$ is defined with an affix $(-q, 0)$ and a radius $r$ such that $(-q+r)<0$. The two scalars $q$ and $r$ are used to determine a specific region included in the left half-plane where the observer eigenvalues will be placed. Therefore, this region puts a lower bound on both the exponential decay rate and the damping ratio of the closed-loop response. The design of the interval observer (4) such that the observer poles are placed in this LMI region requires to find the observer gain $L$ and unknown symmetric matrix $X=X^{T}>0$ such that:

$$
\left(\begin{array}{cc}
-r X & q X+\left((A(\theta)-L C(\theta))^{T} X\right)^{T} \\
\left(q+(A(\theta)-L C(\theta))^{T} X\right. & -r X
\end{array}\right)<0,
$$

for all $\theta \in \Theta$.

Notice that expression (8) is a Bilinear Matrix Inequality (BMI) since there is a product between $L$ and $X$. Thus, it cannot be solved with LMI classical tools. But, it is possible to transform it into the following LMI by means of the following change of variable $W=L^{T} X$,

$$
\left(\begin{array}{cc}
-r X & q X+X^{T} A(\theta)-W^{T} C(\theta)^{T} \\
\left(q+A(\theta)^{T}\right) X-C(\theta)^{T} W & -r X
\end{array}\right)<0 .
$$

Then, the design procedure boils down to solving the LMI (9) and determining $L=\left(W X^{-1}\right)^{T}$.

To practically solve (9), two approaches have been proposed in the literature. The first approach is based on gridding the parameter space and solving the LMI's for each point in the grid. However, this approach does not rigourously guarantee observer performance for all $\theta \in \Theta$. Alternatively, a second approach can be used based on representing the interval model by a polytopic model that includes the interval model. The polytopic model is obtained by the convex hull approach (see [4]). Then, applying the results presented in the same reference [4], the solution of LMIs (9) can be obtained by solving them in the vertices of the polytopic model if the matrix $C$ does not depend on the uncertain parameters $\theta$. In case this matrix depends of the uncertain parameters, [4] proposes a transformation of the system representation by means of including a filter. 


\subsection{Fault detection test}

Fault detection is based on generating a nominal residual comparing the measurements of physical system variables $y(t)$ with their estimation $\hat{y}(t)$ provided by the observer (4):

$$
r(t)=y(t)-\hat{y}\left(t, \theta_{n}\right)
$$

where $r(t) \in \mathfrak{R}^{n_{y}}$ is the residual set and $\theta_{n}$ being the nominal parameters that can be obtained as: $\theta_{n}=(\bar{\theta}+\underline{\theta}) / 2$. According to [25], the computational form of the nominal residual generator, R2-4 obtained using (4), is:

$$
r(s)=\left(I-H\left(s, \theta_{n}\right)\right) y(s)-G\left(s, \theta_{n}\right) u(s)
$$

that has been derived taken into account that the input/output form of the system (1)-(2) (assuming zero initial conditions) is given by

$$
y(s)=y_{0}(s, \tilde{\theta})+G_{f_{a}}(s, \tilde{\theta}) f_{a}(s)+G_{f_{y}}(s, \tilde{\theta}) f_{y}(s)
$$

where:

$$
\begin{aligned}
y_{0}(s, \tilde{\theta}) & =G_{u}(s, \tilde{\theta}) u_{0}(s) \\
G_{u}(s, \tilde{\theta}) & =C(\tilde{\theta})(s I-A(\tilde{\theta}))^{-1} B(\tilde{\theta}) \\
G_{f_{a}}(s, \tilde{\theta}) & =C(\tilde{\theta})(s I-A(\tilde{\theta}))^{-1} F_{a}(\tilde{\theta}) \\
G_{f_{y}}(s, \tilde{\theta}) & =F_{y}(\tilde{\theta})
\end{aligned}
$$

and the input/output form of the observer (4) is expressed as follows

$$
\begin{aligned}
\hat{y}(s, \theta) & =G(s, \theta) u(s)+H(s, \theta) y(s) \\
& =G(s, \theta) u_{0}(s)+H(s, \theta) y(s)+G_{f_{u}}(s, \theta) f_{u}(s)
\end{aligned}
$$

where:

$$
\begin{aligned}
G(s, \theta) & =C(\theta)\left(s I-A_{0}(\theta)\right)^{-1} B(\theta) \\
H(s, \theta) & =C(\theta)\left(s I-A_{0}(\theta)\right)^{-1} L \\
G_{f_{u}}(s, \theta) & =G(s, \theta) F_{u}(\theta)
\end{aligned}
$$

When considering model uncertainty located in parameters, the residual generated by (10) will not be zero, even in a non-faulty scenario. To cope with the parameter uncertainty effect, a passive robust approach based on adaptive thresholding can be used [28, 45, 1, 5]. Thus, using this passive approach, the effect of parameter uncertainty in the residual $r(t)$ (associated to each system output $y(t))$ is bounded by the interval [46]:

$$
r(t) \in[\underline{r}(t), \bar{r}(t)]
$$

where:

$$
\underline{r}(t)=\underline{\hat{y}}(t)-\hat{y}\left(t, \theta_{n}\right) \text { and } \bar{r}(t)=\overline{\hat{y}}(t)-\hat{y}\left(t, \theta_{n}\right)
$$


where $\underline{\hat{y}}(t)$ and $\overline{\hat{y}}(t)$ are the bounds of the system output estimation computed component-wise using the interval observer (4) and obtained according to (7). The residual bounds (22) can be expressed in input-output form using (17) as follows:

$$
\begin{aligned}
\underline{r}(t) & =\min _{\theta \in \Theta} \mathscr{L}^{-1}\{\Delta G(s, \theta) u(s)+\Delta H(s, \theta) y(s)\} \\
\bar{r}(t) & =\max _{\theta \in \Theta} \mathscr{L}^{-1}\{\Delta G(s, \theta) u(s)+\Delta H(s, \theta) y(s)\}
\end{aligned}
$$

where: $\Delta G(s, \theta)=G(s, \theta)-G\left(s, \theta_{n}\right)$ and $\Delta H(s, \theta)=H(s, \theta)-H\left(s, \theta_{n}\right) . \quad \theta_{n} \quad$ are the nominal parameters.

Then, the fault detection test could be based on checking if the residuals satisfy or not the condition given by (21). In case that this condition does not hold, a fault can be indicated.

However, in this paper, an energy based adaptive thresholding technique will be used based not in bounding directly the residual, as discussed above, but instead a residual evaluation function. The residual evaluation function is the residual energy

$$
J(t)=\|r(t)\|_{e}
$$

that can be evaluated either in the time or frequency domain. Then, the fault detection decision is based on the following rule:

$$
\begin{array}{lll}
J(t)<J_{t h}(t) & \text { for } & f(t)=0, \\
J(t)>J_{t h}(t) & \text { for } & f(t) \neq 0 .
\end{array}
$$

where $J_{t h}(t)$ is the threshold for the residual energy taking into account parameter uncertainty and $f(t)$ represents the considered faults.

According to the literature [17], fault detection test based on residual energy evaluation has the advantage that is less sensitive to noise that basic fault detection test (21).

The threshold $J_{t h}$ is generated in such a way that with the considered parameter uncertainty the worst-case energy is bounded. So, while the energy of the nominal residual is less than this threshold no fault can be indicated. Otherwise, a fault can be indicated. This is the way how robustness is achieved. On the other hand, this threshold establishes the minimum detectable fault. That is, the minimum fault size such that will produce a energy bigger that the threshold. Faults smaller than this size will not be detected (missed alarms).

\section{ADAPTIVE THRESHOLDING IN THE TIME DOMAIN}

\subsection{Energy based adaptive thresholding in the time domain}

As discussed in [17], the residual evaluation function based on residual energy $J(t)=\|r(t)\|_{e}$ can be evaluated in the time domain using a time window $T^{\dagger}$ as follows:

${ }^{\top}$ The selection of the time window length will be discussed later in the paper. 


$$
J(t)=\|r(t)\|_{e, t, T}=\|r(t)\|_{2, t, T}=\sqrt{\int_{t-T}^{t} r^{\prime}(\tau) r(\tau) d \tau}
$$

Then, the adaptive threshold $J_{t h}(t)$ in (26) can be computed as:

$$
J_{t h, t}(t)=\max \left(\|\underline{r}(t)\|_{e, t, T},\|\bar{r}(t)\|_{e, t, T}\right)
$$

where

$$
\|\underline{r}(t)\|_{e, t, T}=\|\underline{r}(t)\|_{2, t, T}=\sqrt{\int_{t-T}^{t} \underline{\underline{r}^{\prime}}(\tau) \underline{r}(\tau) d \tau}
$$

and

$$
\|\bar{r}(t)\|_{e, t, T}=\|\bar{r}(t)\|_{2, t, T}=\sqrt{\int_{t-T}^{t} \bar{r}^{\prime}(\tau) \bar{r}(\tau) d \tau}
$$

The interval for residual $[\underline{\hat{r}}(t), \overline{\hat{r}}(t)]$ needed to compute adaptive threshold $J_{t h}(t)$ can be obtained from the interval predicted output $[\underline{\hat{y}}(t), \overline{\hat{y}}(t)]$ by means of (23) and (24). The interval for the predicted output can determined by computing the worst-case time response of the observer given by (4).

The computation of the worst-case time response will require to solve two optimization problems (7) for each output subject to:

$$
\begin{gathered}
\hat{y}_{i}(t, \theta)=C(\theta) e^{A(\theta)\left(t-t_{o}\right)} x\left(t_{o}\right)+\int_{t_{o}}^{t} C(\theta) e^{A(\theta)(t-\tau)} B(\theta) u(\tau) d \tau \\
x(0) \in[\underline{x}(0), \bar{x}(0)]
\end{gathered}
$$

This problem was first formulated by Horak [28] in the context of adaptive threshold generation. He proposed an algorithm called RMI ("reachable minimum interval") based on dynamic optimization to solve it $\mathrm{it}^{\ddagger}$. The difficulty of solving this problem is due to the fact that in general it is not true that considering only the responses at vertices of uncertain parameter and initial conditions intervals would produce the tightest interval containing all possible time responses. Only a small number of systems satisfy this desirable property [26]. In particular, they are those that satisfy the quasi-isotonicity property.

In case that this property is not satisfied, the vertex solution only provides an inner solution, according to Kolev [31]. The existent algorithms to determine $[\hat{y}(t)]$ follow mainly two approaches after applying some kind of time-discretization: the first approach is based on trying to solve the associated optimizations problems (7) (see for example [46]). This is not in general an easy task since global optimization is required to avoid local minima induced by the non-convexity of the problems (7).

\footnotetext{
$\$$ In the literature this problem is found in many places: Qualitative Reasoning [10, 33], Constraint Satisfaction [16], Validated Initial Value Problems [9, 30, 35, 40, 41, 42], Automatic Control [6, 20, 49], Fault Detection[5, 28, 45] and Circuit Tolerance Analysis [23, 31]
} 
On the other hand, the second approach tries to find one step recursion that provides interval $[\hat{y}(t)]$ at present instant from previous intervals determined in previous time instants (see for example [20]). However, in this case there are also some problems as the wrapping effect that must be handled [46]). In this paper, this second approach will be followed by using zonotopes as means to handle the wrapping effect [2]. This approach guarantees that the zonotope computed at each iteration encloses all possible states reached by the interval observer.

\subsection{Implementation using set-computations}

In order to compute the interval $[\hat{y}(t)]$ at present instant from previous intervals determined in previous time instants using zonotopes and the method presented in [2] (see Appendix A), the observer (4) should be discretised. In principle any time discretisation scheme can be used. In this paper, the Euler discretisation approach has been applied what leads the observer (4) to formulated in discrete-time as follows:

$$
\begin{aligned}
& \hat{x}(k)=\tilde{A}_{0}(\theta) \hat{x}(k-1)+\tilde{B}_{0}(\theta) v(k-1) \\
& \hat{y}(k)=\tilde{C}(\theta) \hat{x}(k)
\end{aligned}
$$

where: $\quad \tilde{A}_{0}(\theta)=I+\Delta t(A(\theta)-L C(\theta)), \quad \tilde{B}_{0}(\theta)=\Delta t[B(\theta) L] \quad$ and $\quad v(k)=[u(k) y(k)]^{\prime} \quad$ and $\quad \Delta t$ is discretisation time step.

The discretisation error can also be bounded and included as extra uncertainty in the model in order to guarantee that the interval $[\hat{y}(t)]$ contains all possible trajectories generated from the continuous-time observer (4) at each time instant [30].

Definition 1. Given the sequence of measured inputs $(u(i))_{0}^{k-1}$ and outputs $(y(i))_{0}^{k-1}$ and assuming that the initial states are bounded by a known compact set $\mathbb{X}_{0}$. Then, the exact uncertain estimated state set $\mathbb{X}_{k}$ at time $k$ is expressed by:

$$
\mathbb{X}_{k}=\left\{\hat{x}(k):\left(\hat{x}(i)=\tilde{A}_{0}(\theta) \hat{x}(i-1)+\tilde{B}_{0}(\theta) v(i-1)\right)_{i=1}^{k} \mid \hat{x}_{0} \in \mathbb{X}_{0}, \quad \theta \in \Theta\right\}
$$

The uncertain state set described in Definition 1 at time $k$ can be computed approximately by admitting the rupture of the existing relations between variables of consecutive time instants. This allows to compute an approximation of this set from the approximate uncertain set at time $k-1$.

Definition 2. Consider the interval observer given by (33)-(34), the set of uncertain states at time $k-1\left(\mathbb{X}_{k-1}\right)$ and the input/ouput values $\{u(k-1), y(k-1)\}$. Then, the approximate set of estimated states $\mathbb{X}_{k}^{e}$ at time $\mathrm{k}$ based on the measurements up to time $k-1$ is defined as:

$$
\mathbb{X}_{k}^{e}=\left\{\hat{x}(k): \hat{x}(k)=\tilde{A}_{0}(\theta) \hat{x}(k-1)+\tilde{B}_{0}(\theta) v(k-1) \mid \hat{x}(k-1) \in \mathbb{X}_{k-1}, \quad \theta \in \Theta\right\}
$$

Definition 1 and Definition 2 can be easily adapted to describe the exact uncertain estimated output set $\mathbb{Y}_{k}$ at time $k$ and the approximate set of estimated outputs $\mathbb{Y}_{k}^{e}$. 
Since the exact set of estimated states $\mathbb{X}_{k}^{e}$ and outputs $\mathbb{Y}_{k}^{e}$ are difficult to compute, one way is to bound them using some geometric shapes easy to compute as for example: boxes (interval hull) as in [44], ellipsoids as in [36] or zonotopes as in [2].

Here, the set of estimated states $\mathbb{X}_{k}^{e}$ (or outputs $\mathbb{Y}_{k}^{e}$ ) introduced in Definition 2 will be approximated iteratively using zonotopes (see Appendix A). From these zonotopes, an interval for each state variable and output can also be obtained by computing the interval hull of the zonotope $\mathbb{Z}$, denoted as $\square \mathbb{Z}$. The sequence of interval hulls $\square \mathbb{X}_{k}^{e}$ and $\square \mathbb{Y}_{k}^{e}$ with $k \in[0, n]$ will be called the interval observer estimation of the system (33)-(34) where $n$ is the number of measurement data considered. Following the previous idea, Algorithm 1 is proposed to determine an approximation of set of uncertain estimated states and outputs.

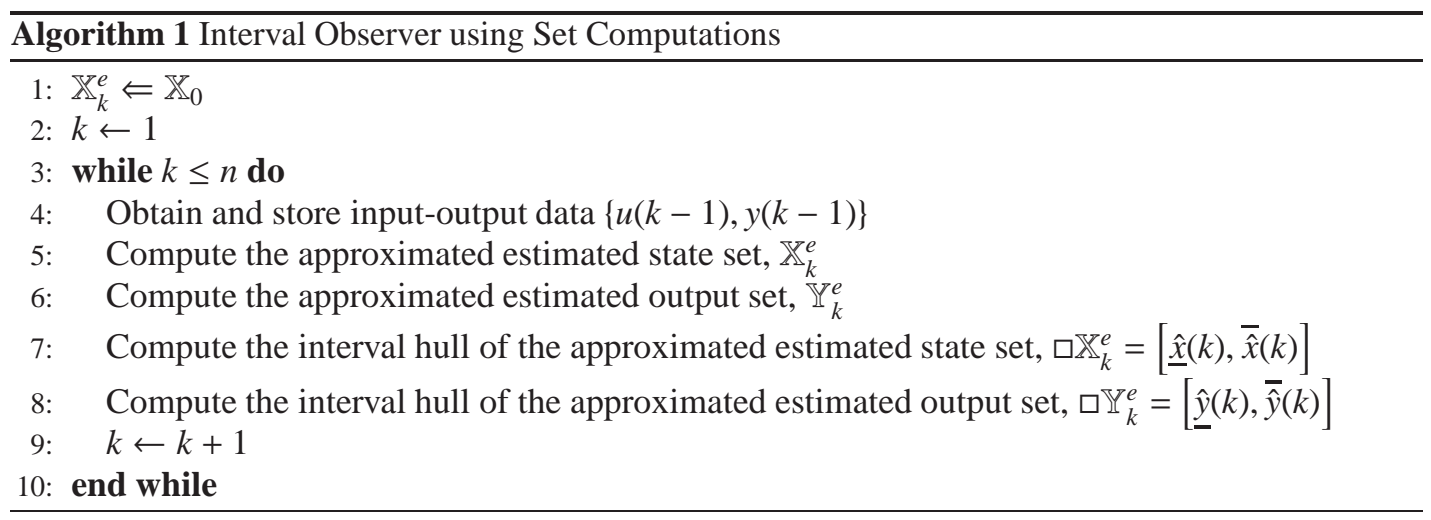

The implementation of steps 5-8 in the Algorithm 1 using zonotopes is described in detail in Appendix A

\section{ADAPTIVE THRESHOLDING IN THE FREQUENCY DOMAIN}

\subsection{Introduction}

Adaptive thresholding in the frequency domain has started with the seminal work of EmamiNaeini [21]. Then, it was followed by Ding [18] and Frank [24]. In these works, the uncertainty was considered unstructured. But, more recently, Rambeaux [47] and Hamelin [27] have considered the case of structured uncertainty. All these works try to bound the residual's energy taking into account the uncertainty in the model. The first approaches considering unstructured uncertainty use $H_{\infty}$ techniques to bound the effect of this uncertainty and the second approaches considering structured uncertainty use instead interval polynomial methods. In the frequency domain approach, although the considered residual evaluation function is again the residual's energy, it is evaluated in the frequency domain according to:

$$
J(t)=\|r(t)\|_{e, \omega, W}=\|r(t)\|_{2, \omega, W}=\sqrt{\frac{1}{\pi} \int_{0}^{W} r^{\prime}(\omega) r^{*}(\omega) d \omega}
$$

where $r(\omega)=\mathcal{F}\{r(t)\}$ and $W$ is the frequency window. 
In order to use (37) in the fault detection test (26), the adaptive threshold $J_{\text {th }}$ should also be obtained in the frequency domain considering the effect of the parametric uncertainty in the residual as follows

$$
J_{t h, \omega}(t)=\sup _{\theta \in \Theta}\left(\frac{1}{\pi} \int_{0}^{W}|r(\omega, \theta)|^{2} d \omega\right)^{\frac{1}{2}}
$$

or alternatively, considering (23)-(24) as follows

$$
J_{t h, \omega}(t)=\sup _{\theta \in \Theta}\left(\frac{1}{\pi} \int_{0}^{W}|\Delta G(\omega, \theta) u(\omega)+\Delta H(\omega, \theta) y(\omega)|^{2} d \omega\right)^{\frac{1}{2}}
$$

This allows to compute the adaptive threshold $J_{t h}$ that will be used in the context of the fault detection rule (26).

\subsection{Energy based adaptive thresholding using $H_{\infty}$}

According to Chen [11], the adaptive threshold $J_{t h}(38)$ can be bounded in the $H_{\infty}$ framework as follows

$$
J_{t h, \omega}(t)<\delta_{u}\|u(t)\|_{e}+\delta_{y}\|y(t)\|_{e}
$$

taking into account that

$$
\|\Delta G(\omega, \theta)\|_{\infty}<\delta_{u} \text { and }\|\Delta H(\omega, \theta)\|_{\infty}<\delta_{y}
$$

for all $\theta \in \Theta$ and where $\|u(t)\|_{e}$ and $\|y(t)\|_{e}$ are the input and ouptut energy, respectively, evaluated at every time instant either in the time domain (27) or in the frequency domain (37).

Then, the fault detection decision rule (26) applies as well.

\subsection{Energy based adaptive thresholding using Kharitonov polynomials}

The $H_{\infty}$ approach bounds the worst-case frequency response in a non-structured way as indicated in (41). Alternatively, the worst-case frequency response can be better approximated in the parametric framework. In particular, the evaluation of $J_{t h}$ through (38) requires to determine

$$
\sup _{\theta \in \Theta}|r(\omega, \theta)|
$$

according to Rambeaux [47]. This problem is related to the evaluation of frequency response envelopes of an interval system. The maximum envelope always corresponds with

$$
\sup _{\theta \in \Theta}|r(\omega, \theta)|=\frac{\sup _{\theta \in \Theta}|N(\omega, \theta)|}{\inf _{\theta \in \Theta}|D(\omega, \theta)|}
$$

and it can be determined using Kharitonov polynomials (see Appendix B) associated with the numerator $N$ and the denominator $D$ of $r$ assuming that the uncertain parameters $\theta$ are independent [34]. However, frequency envelopes are not defined by a single analytic function in all the frequency ranges. Instead, the frequency domain should be decomposed into different frequency intervals $\left[\omega_{k_{i}}, \omega_{l_{i}}\right], i=1, \ldots, p, p$ being the number of intersections of the Kharitonov rectangles with 
both the imaginary and real axes. Hence, the maximum envelope can be expressed as follows:

$$
\sup _{\theta \in \Theta}|r(\omega, \theta)|=\sum_{i=1}^{p}\left|\bar{r}_{i}(\omega)\right| I_{i}
$$

where $\bar{r}_{i}(\omega)=r\left(\omega, \theta_{i}\right)$ is the maximum frequency envelope in the frequency range $\left[\omega_{k_{i}}, \omega_{l_{i}}\right]$ obtained with a given value of the uncertain parameter $\theta_{i}$ and $I_{i}$ is an indicative function such that

$$
I_{i}=\left\{\begin{array}{ccc}
1 & \text { if } & \omega \in\left[\omega_{k_{i}}, \omega_{l_{i}}\right] \\
0 & \text { if } & \omega \notin\left[\omega_{k_{i}}, \omega_{l_{i}}\right]
\end{array}\right.
$$

Then

$$
J_{t h, \omega}=\left(\frac{1}{\pi} \int_{0}^{W} \sum_{i=1}^{p}\left(\left|\bar{r}_{i}(\omega)\right| I_{i}\right)^{2} d \omega\right)^{1 / 2}=\left(\sum_{i=1}^{p} \frac{1}{\pi} \int_{\omega_{k_{i}}}^{\omega_{l_{i}}}\left(\left|\bar{r}_{i}(\omega)\right| I_{i}\right)^{2} d \omega\right)^{1 / 2}
$$

\section{RELATING TIME AND FREQUENCY RESIDUAL EVALUATION APPROACHES}

After presenting the time and frequency domain approaches to residual evaluation and adaptive threshold generation, we now relate these two approaches through Parseval's Theorem [3].

\subsection{Equivalence of unlimited time and frequency residual energy evaluation}

In Fourier signal analysis, the Parseval's Theorem establishes a link between the evaluation of the signal's energy in the time and frequency domains, as follows

$$
\|r(t)\|_{e}=\|r(t)\|_{2, t}=\|r(t)\|_{2, \omega}
$$

where:

$$
\begin{gathered}
\|r(t)\|_{2, t}=\sqrt{\int_{0}^{\infty} r^{\prime}(\tau) r(\tau) d \tau} \\
\|r(t)\|_{2, \omega}=\sqrt{\frac{1}{\pi} \int_{0}^{+\infty} r^{\prime}(\omega) r^{*}(\omega) d \omega}
\end{gathered}
$$

The equivalence between time and frequency signal energy evaluation established by Parseval's Theorem requires that the signal should be evaluated in time-domain in the interval $t \in[0, \infty)$ and in frequency-domain in the interval $\omega \in(-\infty, \infty)$, respectively. However, the residual time evaluation function (27) considers the energy in a limited time and the frequency evaluation function (37) considers energy in a limited frequency window.

\subsection{Equivalence of time and frequency limited residual energy evaluation}

Defining a time-window limited residual as

$$
r_{T}(t)=r(t) i_{[t-T, t]}(t)
$$


with:

$$
i_{\left[t_{0}, t_{1}\right]}(t)=\left\{\begin{array}{lll}
1 & \text { if } & t \in\left[t_{0}, t_{1}\right] \\
0 & \text { if } & t \notin\left[t_{0}, t_{1}\right]
\end{array}\right.
$$

the residual time evaluation function (27) can be written as

$$
\|r(t)\|_{2, t, T}=\sqrt{\int_{t-T}^{t} r^{\prime}(\tau) r(\tau) d \tau}=\sqrt{\int_{0}^{\infty} r_{T}^{\prime}(\tau) r_{T}(\tau) d \tau}=\left\|r_{T}(t)\right\|_{2, t}
$$

Then, applying Parseval's Theorem to (52):

$$
\left\|r_{T}(t)\right\|_{2, \omega}=\sqrt{\int_{0}^{\infty} r^{\prime} T(\tau) r_{T}(\tau) d \tau}=\sqrt{\frac{1}{\pi} \int_{0}^{\infty} r^{\prime} T(\omega) r_{T}^{*}(\omega) d \omega}
$$

where: $r_{T}(\omega)$ is the Fourier Transform of $r_{T}(t)$. Therefore:

$$
\left\|r_{T}(t)\right\|_{2, t}=\left\|r_{T}(t)\right\|_{2, \omega}
$$

Finally, to relate residual time evaluation function (27) with frequency evaluation function (37) $\left\|r_{T}\right\|_{2, \omega}$ should be evaluated over a finite frequency band $W$ :

$$
\left\|r_{T}(t)\right\|_{2, \omega, W}=\sqrt{\frac{1}{\pi} \int_{0}^{W} r_{T}^{\prime}(\omega) r_{T}^{*}(\omega) d \omega}
$$

what is equivalent to band-limiting the residual by a filter of bandwidth $W$.

Notice that in order to the energy evaluation in the time and frequency domains provide equivalent results, both approaches should evaluate the energy of the same signal. Since in the time domain the energy evaluation is done over a time-window limited residual $r_{T}$, the same signal should also be considered in the frequency domain evaluation. Otherwise, if the whole residual $r$ is used in the frequency domain, the resulting energy will be larger.

Thus, bandlimiting the residual $r_{T}$ as follows

$$
r_{T, W}(\omega)=r_{T}(\omega) I_{[0, W]}(\omega)
$$

with:

$$
I_{[0, W]}(\omega)=\left\{\begin{array}{lll}
1 & \text { if } & \omega \in[0, W] \\
0 & \text { if } & \omega \notin[0, W]
\end{array}\right.
$$

then:

$$
\left\|r_{T, W}(t)\right\|_{2, \omega}=\sqrt{\frac{1}{\pi} \int_{0}^{+\infty} r_{T, W}^{\prime}(\omega) r_{T, W}^{*}(\omega) d \omega}
$$

where $r_{T, W}(\omega)$ is the Fourier Transform of $r_{T}(t)$ band-limited to a bandwidth $W$. Finally, according to Parseval's Theorem applied to (58):

$$
\left\|r_{T, W}(t)\right\|_{2, \omega} \approx\left\|r_{T, W}(t)\right\|_{2, t}=\sqrt{\int_{t-T}^{t} r_{T, W}^{\prime}(\tau) r_{T, W}(\tau) d \tau}
$$


So, residual evaluation in time and frequency domain using, respectively, (27) and (37), would be equivalent if residual energy is evaluated, at the same time, in a finite time window $T$ and frequency band $W$. However, as it is known from signal theory, according to the time limited-band limited theorem, no signal can be both time limited and band limited simultaneously. This is because timelimiting a signal is equivalent in the frequency to convolute its spectrum with a "sinc" signal whose spectrum is of infinite extent. Analogously, band-limiting a signal is equivalent in the time-domain to convolute it with a time "sinc" signal whose extent is also infinite. This theorem implies that every signal must be of infinite extent, either in time or frequency domains, or in both domains. Therefore, equality (58) will only hold for a residual time-limited to a time horizon $T$ if the bandwidth $W$ tends to $\infty$, or alternatively, for a residual band-limited to a band $W$ if the time horizon $T$ tends to $\infty$. As a conclusion, the equivalence of residual evaluation functions (27) and (37) require, either an infinite time horizon or bandwidth to be equivalent. However, from the practical point of view, time and frequency domain energy evaluation using a time-limited residual $r_{T}$ would lead to very close results if the time $T$ and the frequency window $W$ are taken long enough. This will be seen in the following example.

\subsection{An example}

The example is based on that proposed by [19] and will be used in order to compare time and frequency domain residual evaluation approaches.

Let us consider that the expression for the residual to be evaluated is:

$$
r(s)=\frac{1}{\alpha s+1}
$$

Or, alternatively in time domain:

$$
r(t)=\frac{1}{\alpha} e^{-\frac{t}{\alpha}}
$$

if $t \geq 0$ and $r(t)=0$ if $t<0$.

5.3.1. Energy evaluation in the time domain. Applying residual evaluation function in the timedomain (27) using a time window $T$ :

$$
\|r(t)\|_{e}=\|r(t)\|_{2, t, T}=\sqrt{\int_{t-T}^{t} r^{\prime}(\tau) r(\tau) d \tau}=\sqrt{\frac{1}{2 \alpha}\left[\left(1-e^{-2 \frac{t}{\alpha}}\right) u(t)-\left(1-e^{-2 \frac{t-T}{\alpha}}\right) u(t-T)\right]}
$$

Figure 1 shows how the residual energy evaluation varies with the time window length $T$. If this length tends to infinity such that the limits of the integral are in the interval $t \in[0, \infty)$ as in Parseval's Theorem:

$$
\|r(t)\|_{e}=\|r(t)\|_{2, t}=\sqrt{\int_{0}^{\infty} r^{\prime}(\tau) r(\tau) d \tau}=\frac{1}{\sqrt{2 \alpha}}
$$

However, from the practical point of view it can be seen that using a time window length $T=3 \alpha$ the value achieved is very close to the one achieved with the infinite window length. 


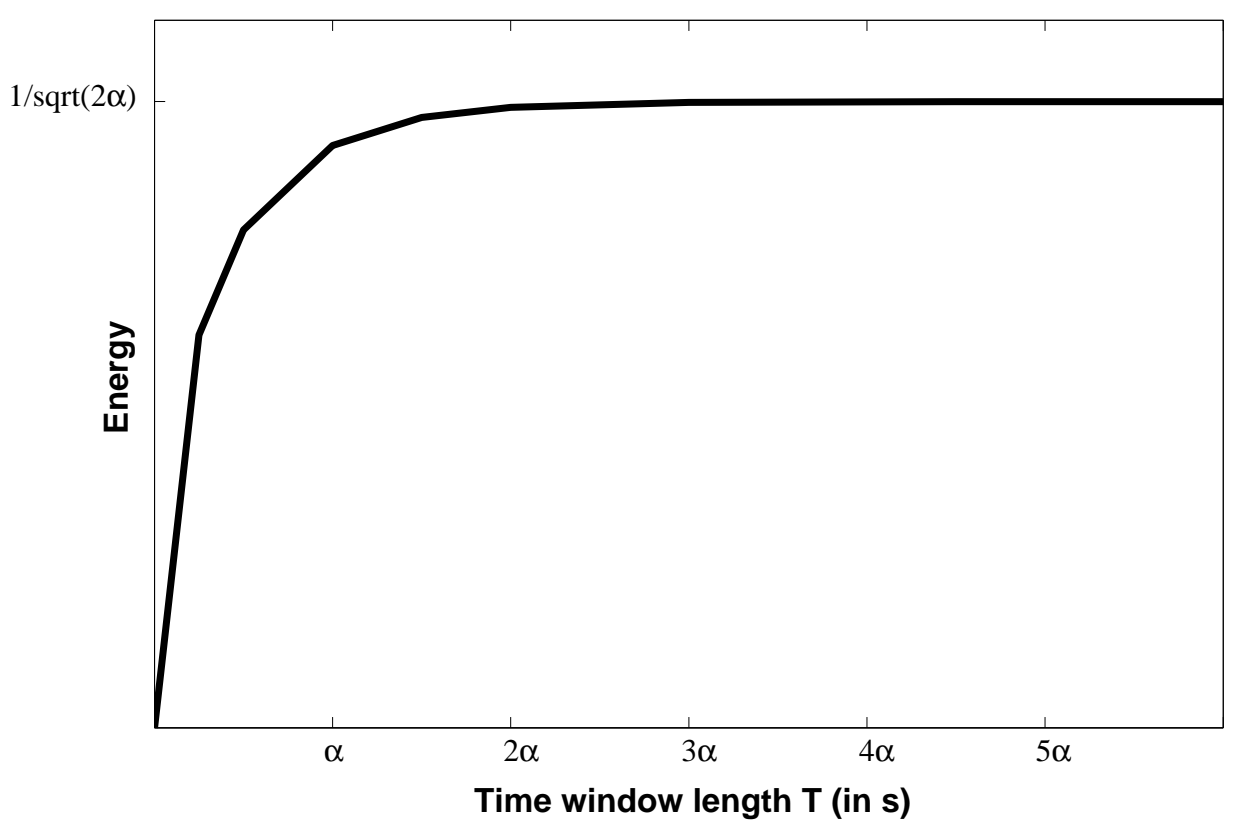

Figure 1. Effect of finite time window length in the residual energy evaluation

5.3.2. Energy evaluation in the frequency domain. Analogously, applying evaluation function (10) in frequency domain:

$$
\|r(t)\|_{e}=\|r(t)\|_{2, \omega, W}=\sqrt{\frac{1}{\pi \alpha} \int_{0}^{W} r^{\prime}(\omega) r^{*}(\omega) d \omega}=\sqrt{\frac{1}{\pi \alpha} \tan ^{-1}(\alpha W)}
$$

assuming that the signal $r(t)$ is known in the time interval: $t \in[0, \infty)$.

Figure 2 shows how the residual energy evaluation varies with the frequency window length $W$. If this length tends to infinity

$$
\|r\|_{e}=\|r\|_{2, \omega, W}=\sqrt{\frac{1}{\pi \tau} \int_{0}^{\infty} r^{\prime}(\omega) r^{*}(\omega) d \omega}=\frac{1}{\sqrt{2 \alpha}}
$$

that corresponds to the result obtained when infinite time windows length is used in (63) which is not surprising due to Parseval's Theorem.

However, from the practical point of view it can be seen that using a frequency window length $W=30 / \alpha$ the value achieved is very close to the one achieved with the infinite window length.

5.3.3. Comparison of energy evaluation in the time and frequency domains. If energy residual evaluation in frequency domain (10)) is applied to the same time windowed residual used in (62), the following result is obtained (assuming than the bandwidth $W$ is infinity or long enough) in order to be comparable with $(62))^{\S}$ :

$\S_{\S}$ Otherwise, a band-limited version of the residual should used in computing (62) in order to obtain the same results in frequency and time domain according to (59). 


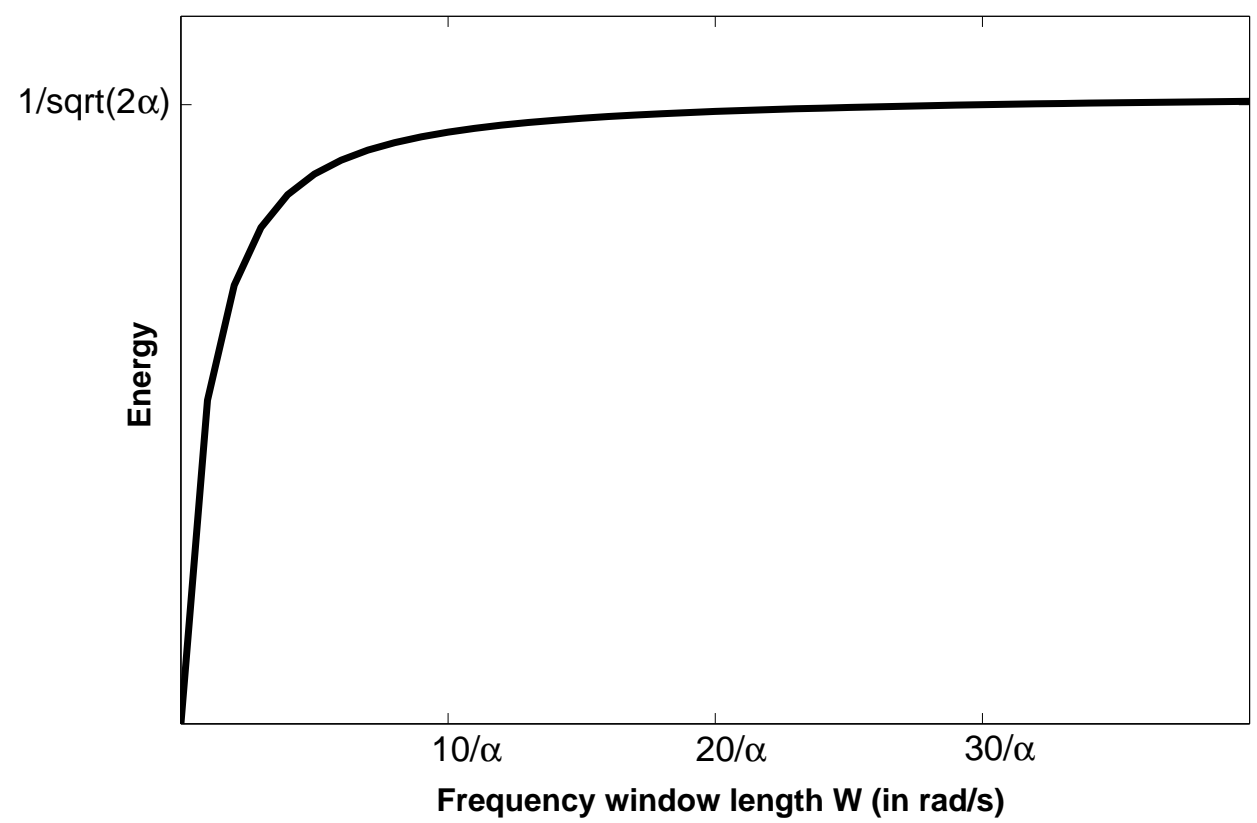

Figure 2. Effect of finite frequency window length in the residual energy evaluation

- for $t<T$ :

$$
\begin{aligned}
\|r(t)\|_{e}=\|r(t)\|_{2, \omega, W} & =\sqrt{\frac{1}{\pi} \int_{0}^{\infty} r_{T}^{\prime}(\omega) r_{T}^{*}(t)(\omega) d \omega} \\
& =\sqrt{\frac{1}{2 \alpha}\left(1-e^{-\frac{2 t}{\alpha}}\right)(u(t)-u(t-T))}
\end{aligned}
$$

where: $r_{T}(\omega)=\int_{0}^{T} r(\tau) e^{-j \omega \tau} d \tau$.

- for $t>T$ :

$$
\begin{aligned}
\|r(t)\|_{e}=\|r(t)\|_{2, \omega, W} & =\sqrt{\frac{1}{\pi} \int_{0}^{\infty} r_{T}^{\prime}(\omega) r_{T}^{*}(\omega) d \omega} \\
& =\sqrt{\frac{1}{2 \alpha}\left(e^{-2 \frac{t-T}{\alpha}}-e^{-2 \frac{t}{\alpha}}\right) u(t-T)}
\end{aligned}
$$

where: $r_{T}(\omega)=\int_{t-T}^{t} r(\tau) e^{-j \omega \tau} d \tau$

Figure 3 represents graphically the time domain energy evaluation (62) with a time window length $T=3 \alpha$ and the frequency domain energy evaluation (67) with a frequency window length $W=$ $30 / \alpha$. These window lengths provide similar results that the infinite ones as discussed previously and it can be seen from Figures 1 and 2. From Figure 3, it can be noticed that the same curves are obtained.

Obviously, expressions (66)-(67) tend to (63) if the time window used to evaluate the residual energy in the frequency domain tends to infinity.

Remark. From this example, the following rules of thumb can be used to select the time and frequency windows ( $T$ and $W$, respectively):

- Time window $T$ can be chosen as three times the dominant time constant of the residual (see Figure 1). 

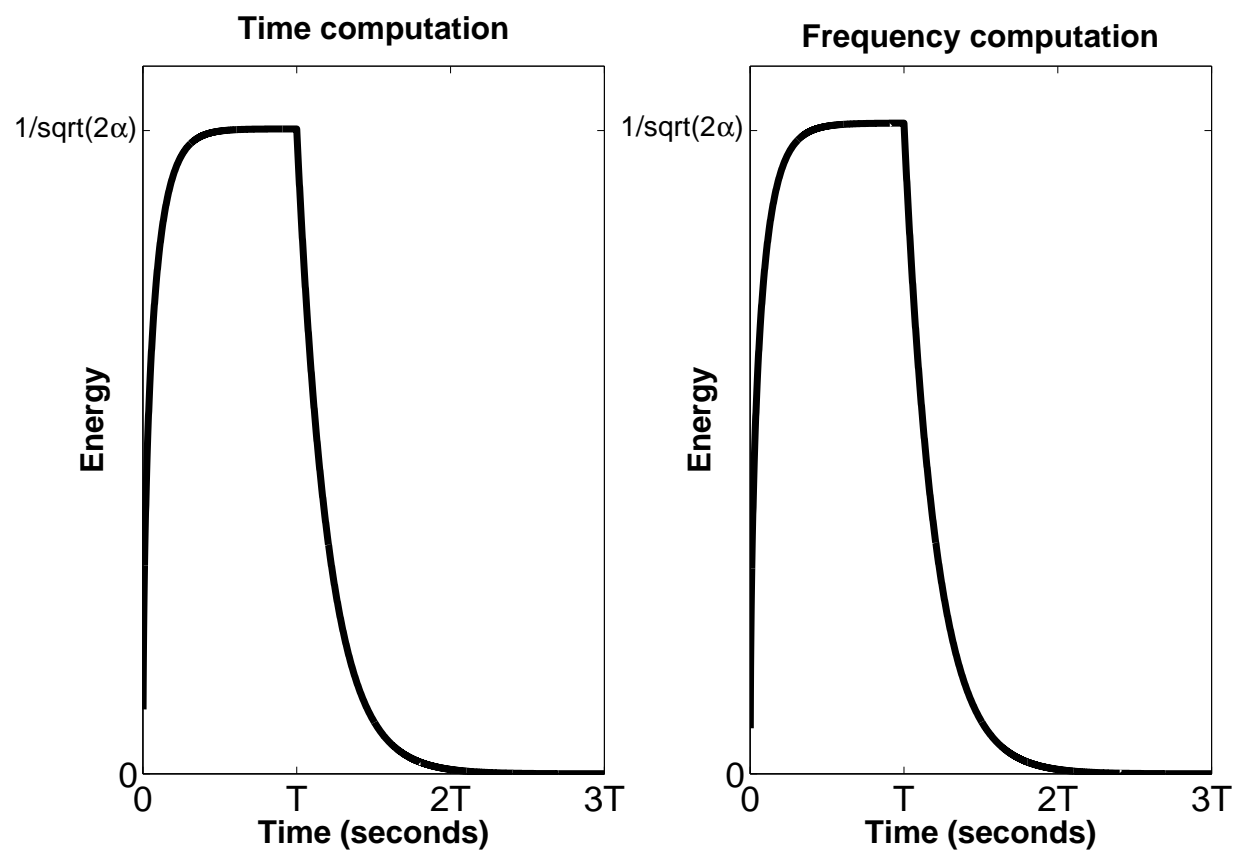

Figure 3. Time vs frequency residual energy computation

- Frequency window $W$ can be chosen as thirty times the inverse of the dominant time constant of the residual (see Figure 2).

Both rules can also be verified analytically for this example by means of Eq. (62) and Eq. (64). For a general residual, the dominant time constant can be extracted, for example, through the spectral analysis determining the bandwidth.

\section{RELATING TIME AND FREQUENCY ADAPTIVE THRESHOLD GENERATION APPROACHES}

\subsection{Equivalence of time and frequency unlimited adaptive threshold generation}

Again, thanks to Parseval's Theorem, threshold generation for residual energy can be computed either in time and frequency domains

$$
J_{t h}=J_{t h, t}=J_{t h, \omega}
$$

Taking into account that $J_{t h, t}$ and $J_{t h, \omega}$ are given respectively by (27) and (46), this implies

$$
\sup _{\theta \in \Theta}\left(\int_{0}^{+\infty} r^{\prime}(\tau) r(\tau) d \tau\right)^{1 / 2}=\left(\int_{0}^{+\infty} \bar{r}^{\prime}(\tau) \bar{r}(\tau) d \tau\right)^{1 / 2}=\left(\sum_{i=1}^{p} \frac{1}{\pi} \int_{\omega_{k_{i}}}^{\omega_{l_{i}}}\left(\left|\bar{r}_{i}(\omega)\right| I_{i}\right)^{2} d \omega\right)^{1 / 2}
$$

where $\bar{r}(\tau)$ denotes the maximum time envelope of the residual. 
On the other hand, applying Parseval's Theorem to (46) follows

$$
J_{t h}=\left(\sum_{i=1}^{p} \frac{1}{\pi} \int_{\omega_{k_{i}}}^{\omega_{l_{i}}}\left|\bar{r}_{i}(\omega)\right|^{2} I_{i} d \omega\right)^{1 / 2}=\left(\sum_{i=1}^{p} \int_{-\infty}^{\infty} \bar{r}_{i}^{2}(\tau) d \tau\right)^{1 / 2}
$$

Then, the maximum time envelope of the residual can be obtained through

$$
\bar{r}(t)=\left(\sum_{i=1}^{p} \bar{r}_{i}^{2}(t)\right)^{1 / 2}=\left(\sum_{i=1}^{p}\left[\mathcal{F}^{-1}\left\{\bar{r}_{i}(\omega) I_{i}\right\}\right]^{2}\right)^{1 / 2}
$$

Moreover:

$$
\bar{r}_{i}(t)=\mathcal{F}^{-1}\left\{\bar{r}_{i}(\omega) I_{i}\right\}=r\left(t, \theta_{i}\right) \otimes\left(\frac{\omega_{k_{i}}}{\pi} \operatorname{sinc}\left(\frac{\omega_{k_{i}} t}{\pi}\right)-\frac{\omega_{l_{i}}}{\pi} \operatorname{sinc}\left(\frac{\omega_{l_{i}} t}{\pi}\right)\right)
$$

where:

$$
r\left(t, \theta_{i}\right)=\mathcal{F}^{-1}\left\{\bar{r}_{i}(\omega)\right\}
$$

\subsection{Equivalence of time and frequency limited adaptive threshold generation}

The equivalence presented in previous section assumes infinite time and frequency windows. As in the case of residual energy evaluation (see Section 5), using a time window $r_{T}$, the adaptive threshold generation in time domain (28) and frequency domain (46) would be equivalent if a large enough frequency band $W$ is used.

\section{APPLICATION EXAMPLE 1: DAMADICS SERVOACTUATOR}

\subsection{Description}

The first application example is based on the DAMADICS servoactuator proposed as fault detection and isolation benchmark by [8] (see Fig. 4). This actuator modifies the position of a valve to control the flow.

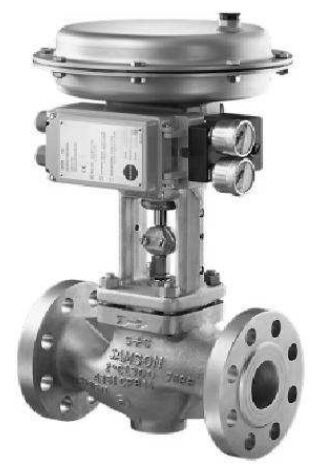

Figure 4. View of the typical industrial control valve actuator.

The servoactuator consists of three main components (see Fig. 5): control valve, spring-anddiaphragm pneumatic servo-motor and positioner [8]. Control valve is used to manipulate the flow. 
The position of the control valve is changed by a servomotor. A spring-and-diaphragm pneumatic servomotor is a compressible (air) fluid powered device in which the fluid acts upon the flexible diaphragm, to provide linear motion of the servomotor stem. Positioner is a device applied to eliminate the control-valve-steam miss-positions produced by the external or internal sources such as friction, pressure unbalance, hydrodynamic forces, etc. It consists in a inner loop with a PI controller of a cascade control structure, including the output signal of the outer loop of the flow or level controller and the inner loop of the position controller.

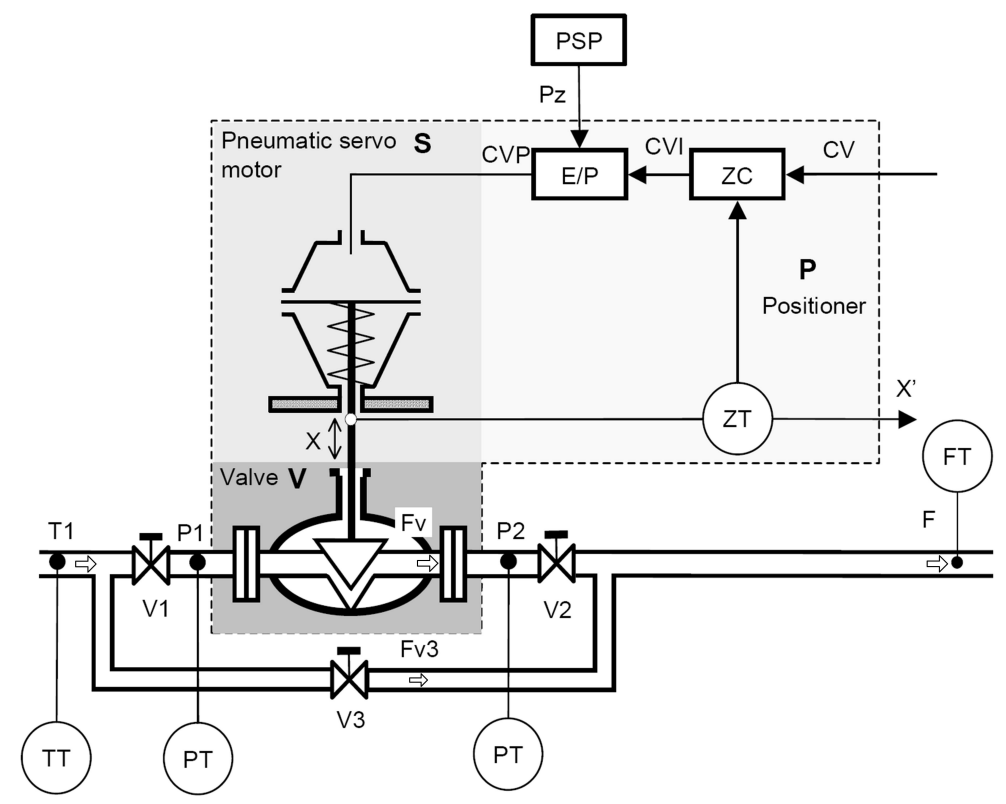

Figure 5. The actuator scheme.

\subsection{Model of DAMADICS servoactuator}

Using physical modelling presented in [8] and the set-membership parameter estimation algorithm proposed in [43], the following linear interval model for the servoactuator has been obtained as:

$$
\begin{aligned}
& \dot{x}(t)=A(\theta) x(t)+B(\theta) u(t) \\
& y(t)=C x(t)
\end{aligned}
$$

where:

$$
A(\theta)=\left(\begin{array}{ccc}
0 & 0 & -\theta_{3} \\
1 & 0 & -\theta_{2} \\
0 & 1 & -\theta_{1}
\end{array}\right), \quad B(\theta)=\left(\begin{array}{l}
\theta_{4} \\
0 \\
0
\end{array}\right), \quad C=\left(\begin{array}{lll}
0 & 0 & 1
\end{array}\right),
$$

$x(t)=\left(x_{1}(t) x_{2}(t) x_{3}(t)\right)^{\prime}$ corresponds to the acceleration, velocity and position of the servomotor, respectively, $u(t)=C V P(t)$ and $y(t)$ is the position of the valve measured by the displacement transducer (in Volt), $C V P(k)$ is the command pressure (in Pascal). The uncertain parameters are bounded by: $\theta_{1} \in[22.13,61.37], \theta_{2} \in[17.33,48.08] \theta_{3} \in[0.388,1.077]$ and $\theta_{4} \in[-2.11,2.3]$ where the nominal values are $\theta_{1}^{0}=41.75, \theta_{2}^{0}=32.71, \theta_{3}^{0}=0.7324$ and $\theta_{4}^{0}=0.0937$. 


\subsection{Application of the fault detection approaches}

Using this interval model (74), a Luenberger interval observer, given by (4), is designed by solving the LMI problem (9) with poles placed in a disk region with affix $q=60$ and radius $r=55$. In this case, since matrix $C$ does not contain uncertain parameters, the LMI problem (9) need only to be solved at the vertices of the parameter intervals, as discussed in Section 2.2. The resulting observer gain is:

$$
L^{\prime}=10^{3}\left[\begin{array}{lll}
6.5669 & 1.3957 & 0.0463
\end{array}\right]
$$

After the interval observer has been designed, the proposed fault detection approaches can be applied as follows: the nominal residual can be generated using nominal parameters according to (10). The interval for the residual that bounds the effect of parameter uncertainty is computed using (21) and Algorithm 1 that computes the interval for observer output estimation. Energy of nominal residual $J$ in the time domain is computed using (27) while in the frequency domain is computed using (37). Residual energy threshold $J_{t h}$ in the time domain is computed using (28) and residual interval (21). On the other hand, this threshold in the frequency domain is computed using (38) and Algorithm 2 to evaluate the worst-case frequency response.

Analyzing the dynamics and frequency response of the residual as it was done in the case of the example presented in Section 5.3, it has been determined that using a time window $T=100 \mathrm{~s}$ and a frequency window $W=2 \mathrm{~Hz}$, the residual and threshold energy evaluation produces results that are equivalent from the practical point of view. This will be illustrated in the following section.

\subsection{Fault scenarios}

The proposed fault detection approaches will be used in two of the fault scenarios proposed in the DAMADICS benchmark: $f_{7}$ (Medium evaporation) and $f_{10}$ (diaphragm perforation). The results are presented in the following.

\subsubsection{Fault $_{7}$ : Medium evaporation}

In this case, a fault in the control valve is introduced (see Fig. 5). The fault is "medium evaporation or critical flow" that consists in two phase flow (mixture of fluid and steam) caused when local fluid pressure drops down to steam evaporation pressure level [7]. In the DAMADICS benchmark, this fault is named as $f_{7}$. In the present experiments, the fault scenario that will be used corresponds to the abrupt big size. The fault appears at time instant $t=900$.

In Fig. 6(a), results from fault detection based on the basic residual evaluation presented in Section 2.2 are presented. The residual signal corresponding to the position of valve is generated and evaluated using the interval for the residual that considers the parametric uncertainty. The interval for the residual is generated using the worst-case time response using zonotopes (81) introduced in Section 3. This fault detection test (26) does not detect the fault in a persistent way after the fault appearance. In Fig. 6(b), results from the evaluation of the residual energy against the adaptive energy threshold $\left(J_{t h}\right)$ are presented when a window time $T=100 \mathrm{~s}$ and a frequency window $W=2 \mathrm{~Hz}$ are used. Applying the fault detection test (26), in the time and in the frequency domain, the fault is detected at $t=900.5 \mathrm{~s}$ until $t=1002 \mathrm{~s}$ corresponding with the end of the time window. Notice than with this time/frequency window lengths, residual and threshold energy evaluation in 
time and frequency domains provides results close enough to be distinguished when represented in the same plot. These results illustrate the equivalences discussed in Section 5 and 6.

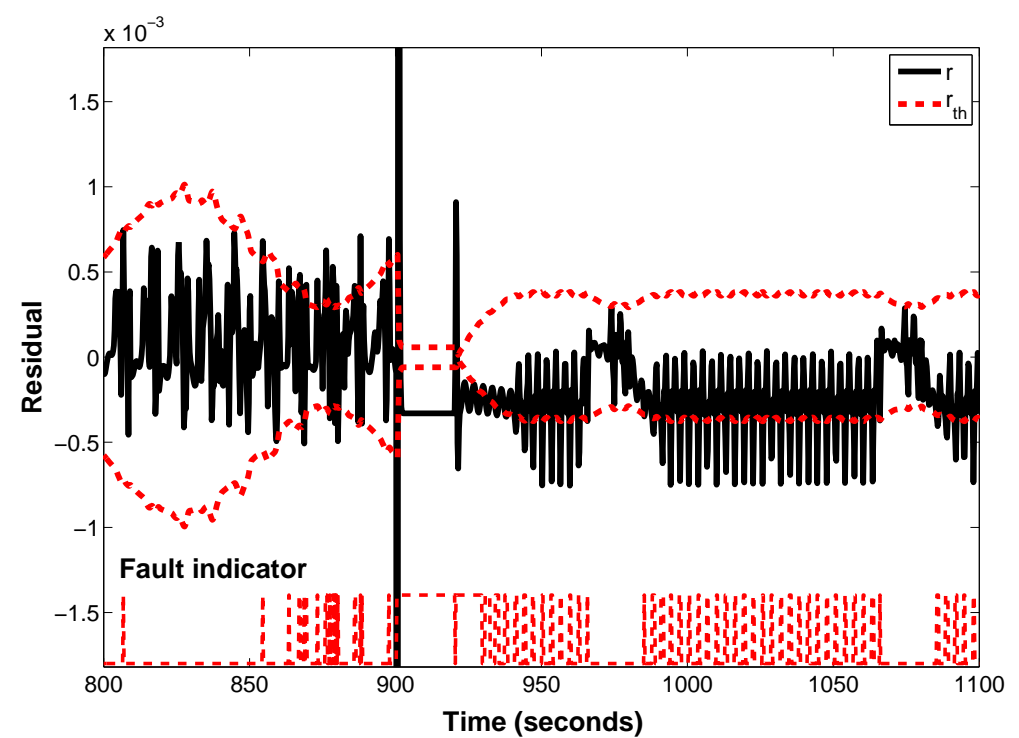

(a)

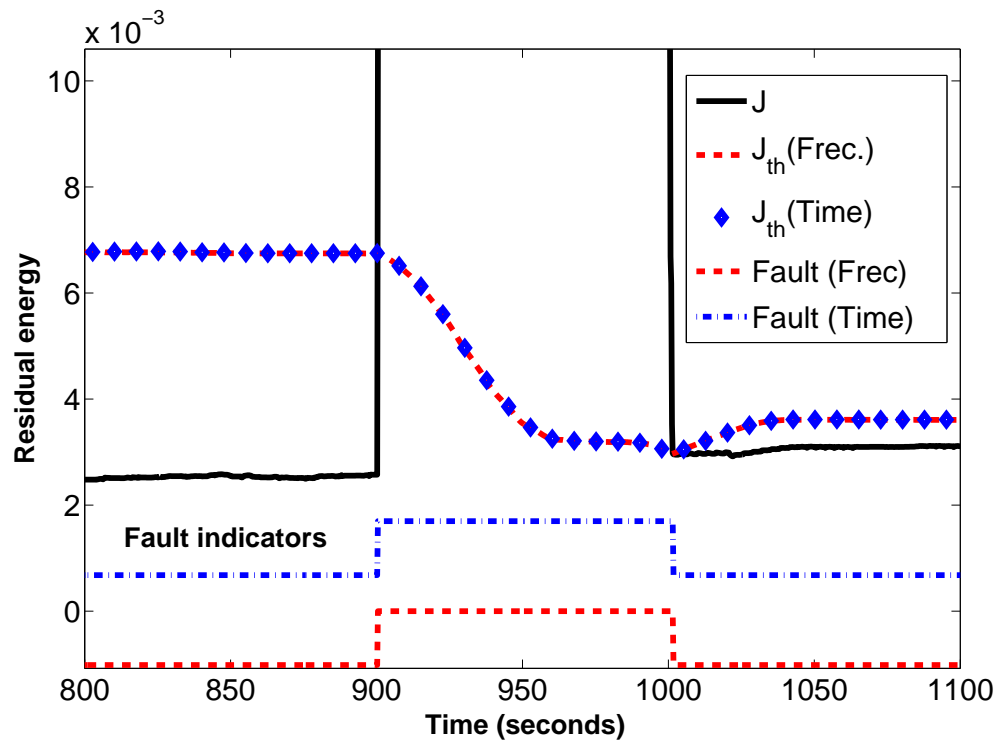

(b)

Figure 6. Fault of medium evaporation or critical flow $f_{7}$. (a) Residual of the critical flow.

(b) Residual energy of the critical flow

\subsubsection{Fault $f_{10}$ : diaphragm perforation}

In this case, a fault in the pneumatic servomotor is introduced (see Fig. 5). The fault consists in servomotor's diaphragm perforation caused by fatigue of diaphragm material [7]. In the DAMADICS benchmark this fault is named as $f_{10}$. In the present experiment, the fault scenario that will be used corresponds to the abrupt big size. The fault appears at time instant $t=900$. 
In Fig. 7(a) results from fault detection based on the basic residual evaluation. As in the case of the fault scenario $f_{7}$, this fault detection test (26) does not detect the fault in a persistent way after the fault appearance. Finally, Fig. 7(b) show the evaluation of the residual energy against the adaptive energy threshold $\left(J_{t h}\right)$ in the time and frequency domains when the same time/frequency windows used in previous fault scenario are used. These results confirm as well the equivalences between the time and frequency approaches presented in Section 5 and 6.

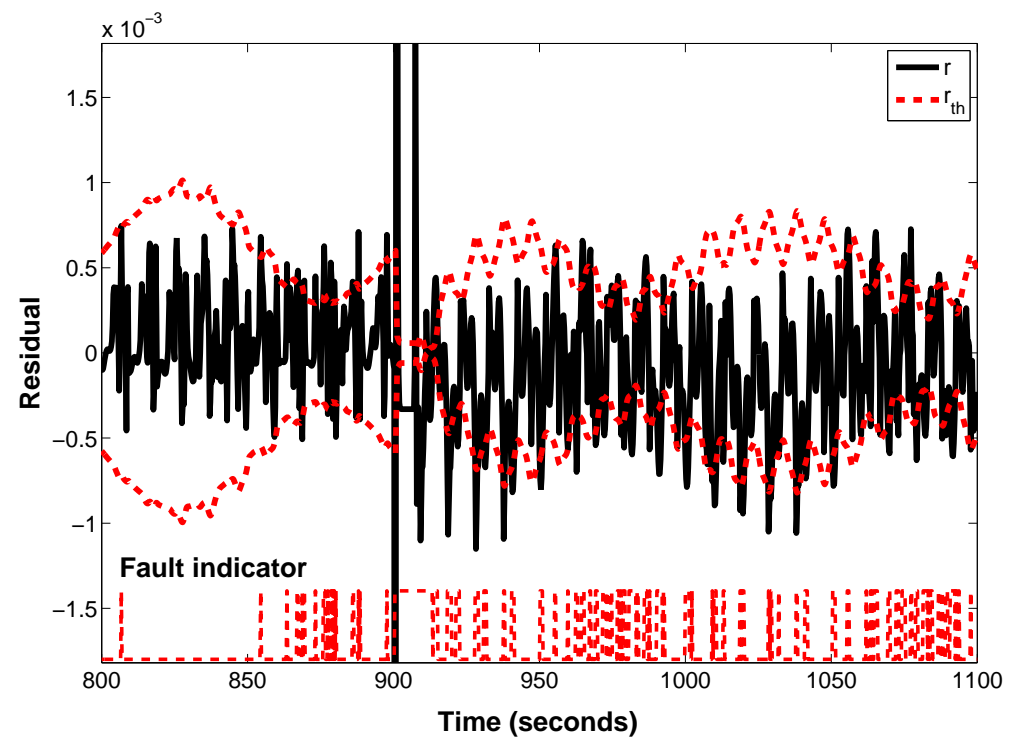

(a)

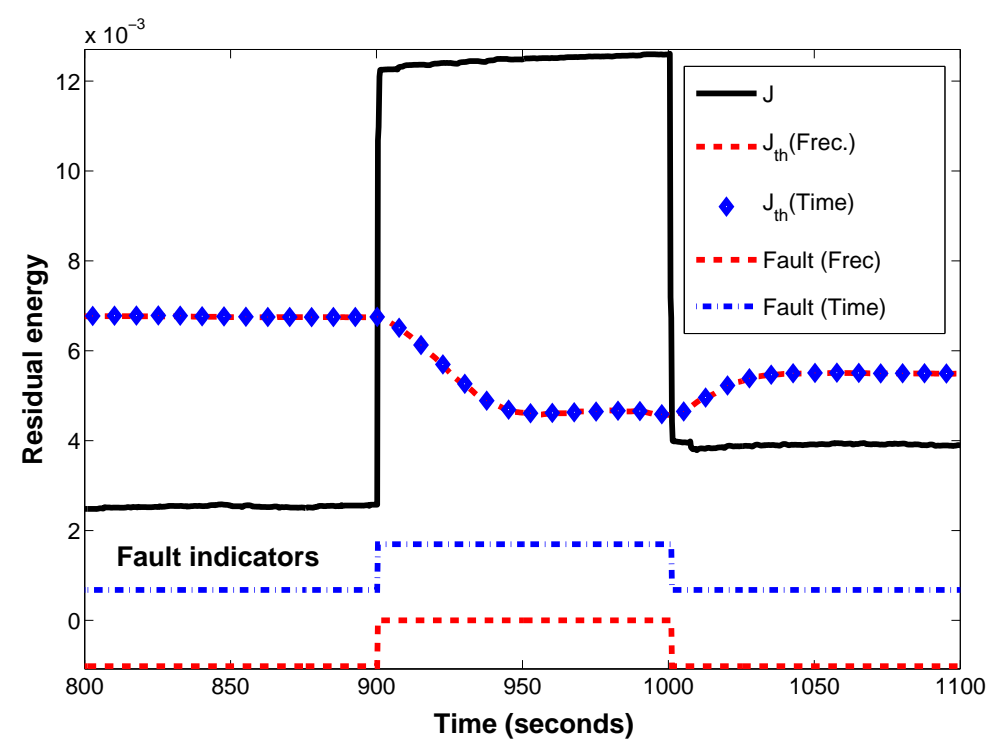

(b)

Figure 7. Fault in the pneumatic servomotor $f_{10}$ (a) Residual of the position of the valve. (b) Residual energy of of the position of the valve. 


\section{APPLICATION EXAMPLE 2: TWIN-ROTOR MIMO SYSTEM}

\subsection{Description of Twin-Rotor MIMO System}

This equipment called TRMS (Twin-Rotor Multiple Input Multiple Output (MIMO) System) is a laboratory set-up developed by Feedback Instruments Limited [22]) available at the laboratories of the Advanced Control Systems Research Group at the Automatic Control Department (ESAII) of Technical University of Catalonia (UPC). The system is perceived as a challenging engineering problem due to its high non-linearity and inaccessibility of some of its states through measurements. The TRMS mechanical unit has two rotors placed on a beam together with a counterbalance whose arm with a weight at its end is fixed to the beam at the pivot and it determines a stable equilibrium position (see Fig. 8). The TRMS consists of a beam pivoted on its base in such a way that it can rotate freely both in the horizontal and vertical planes. At both ends of the beam there are rotors (the main and tail rotors) driven by DC motors.

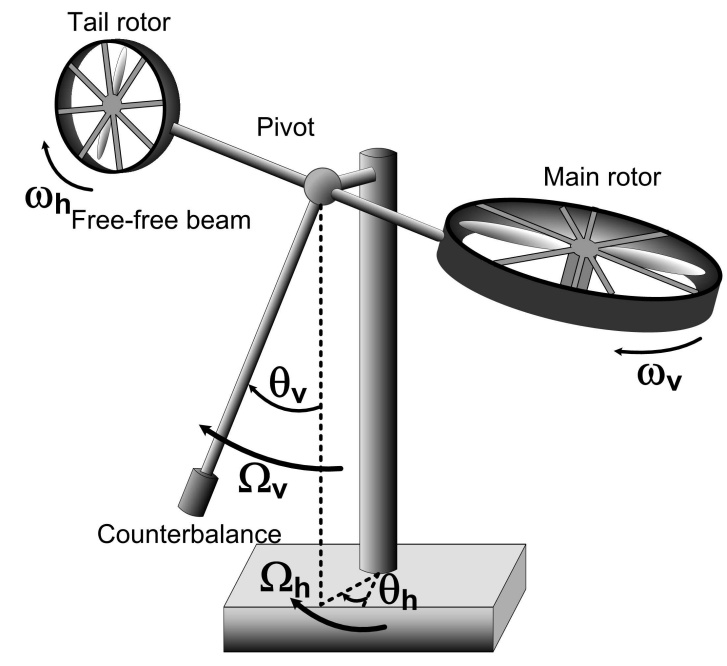

Figure 8. Components of the TRMS

The system input vector is $u=\left(u_{h}, u_{v}\right)^{\prime}$ where $u_{h}$ is the input voltage of the tail motor and $u_{v}$ is the input voltage of the main motor. On the other hand, the system states are $x=$ $\left(\theta_{h}, \Omega_{h}, \theta_{v}, \Omega_{v}, \omega_{t}, \omega_{m}\right)^{\prime}$ where $\Omega_{h}$ is the angular velocity around the vertical axis, $\theta_{h}$ is the azimuth angle of beam (horizontal plane), $\omega_{t}$ is the rotational velocity of the tail rotor, $\Omega_{v}$ is the angular velocity around the horizontal axis, $\theta_{v}$ is the pitch angle of beam (vertical plane) and $\omega_{m}$ is the rotational velocity of the main rotor.

\subsection{Model of TRMS}

A linear model can be obtained by linearisation of the TRMS non-linear model proposed in [22] considering the following input $u=\left(u_{h}, u_{v}\right)^{\prime}$ and output $y=\left(\alpha_{h}, \alpha_{v}\right)^{\prime}$ vectors:

$$
\begin{aligned}
& \dot{x}(t)=A(\theta) x(t)+B(\theta) u_{0}(t) \\
& y(t)=C x(t)
\end{aligned}
$$


where:

$$
\begin{gathered}
A(\theta)=\left(\begin{array}{cccccc}
\theta_{1} & -0.2094 & 3.6941 & 0 & 0 & 0 \\
0 & -0.6863 & 0 & 0 & 0 & 0 \\
0 & 0 & -2.4406 & 0 & 0 & 0 \\
0 & \theta_{2} & -0.5083 & -0.1833 & -3.4363 & 0 \\
0 & 0.0076 & 2.734 & 0.9961 & -0.086 & 0 \\
0.9906 & 0.551 & 0.0947 & 0 & 0 & 0
\end{array}\right), \\
B(\theta)=\left(\begin{array}{cccc}
0.0947 & -0.0053 \\
0 & \theta_{3} \\
0.9377 & 0 \\
-0.0109 & 0.0076 \\
0.0699 & 0.0001 \\
0.0016 & 0.0139
\end{array}\right), \\
C=\left(\begin{array}{cccccc}
0 & 0 & 0 & 0 & 0 & 1 \\
0 & 0 & 0 & 0 & 1 & 0
\end{array}\right),
\end{gathered}
$$

and the following parameters are bounded by: $\theta_{1} \in[-0.5737,-0.1793], \theta_{2} \in[0.1235,0.4835]$ and $\theta_{3} \in[0.9027,1.0627]$ to take into account their variation with the operating point. Their nominal values are $\theta_{1}^{0}=-0.3765, \theta_{2}^{0}=0.3035$ and $\theta_{3}^{0}=0.9827$.

The system is controlled using a MPC (Model Predictive Control) controller designed by the MPC Toolbox of MATLAB with prediction horizon equal to $5 s$ and control horizon equal to $1 s$.

\subsection{Application of the fault detection approaches}

Using this interval model (75), a Luenberger interval observer, given by (4), is designed by solving the LMI problem (9) with poles placed in the disk region with affix $q=27.5$ and radius $r=25$. In this case, as in the DAMADICS example, since matrix $C$ does not contain uncertain parameters, the LMI problem (9) need only to be solved at the vertices of the parameter intervals, as discussed in Section 2.2. The resulting observer gain is:

$$
L^{\prime}=10^{3}\left[\begin{array}{cccccc}
1.3520 & -1.9255 & -0.0158 & -0.0213 & -0.0022 & 0.0374 \\
-0.3927 & 0.6010 & 0.0023 & 0.1742 & 0.0340 & -0.0019
\end{array}\right]
$$

Analyzing the dynamics and frequency response of the residual as it was done in the case of the example presented in Section 5.3, it has been determined that using a time window $T=20 \mathrm{~s}$ and a frequency window $W=20 \mathrm{~Hz}$, the residual and threshold energy evaluation produces results that are equivalent from the practical point of view. This will be illustrated in the following section.

The procedure to apply the proposed fault detection approaches in this example is the same that the one described in previous DAMADICS example (see Section 7.3).

\subsection{Fault scenarios}

The proposed fault detection approaches will be used in three fault scenarios. The results are summarized in the following. 
8.4.1. Fault scenario 1 presents an additive sensor fault of the input voltage of the tail motor $f_{u_{t}}$ :

$$
f_{u_{t}}(t)=\left\{\begin{array}{ccc}
0 V, & \text { for } & t<50 \\
0.12 V, & \text { for } & t \geq 50
\end{array}\right.
$$

In Fig. 9(a)-(b) results from fault detection based on the basic residual evaluation are presented for the azimuth angle and pitch angle of the beam. This fault detection test (21) does not detect the fault in a persistent way after the fault appearance. In Fig. 9(c)-(d) results from the evaluation of the residual energy against the adaptive energy threshold $\left(J_{t h}\right)$ are presented when a time window of $T=20 \mathrm{~s}$ and frequency window of $W=20 \mathrm{~Hz}$ is used. The fault detection test (26) in frequency domain provides equivalent results than the time domain. These results are summarized for azimuth and pitch angle in the Table I, where the detection time instant is presented for each detection test.

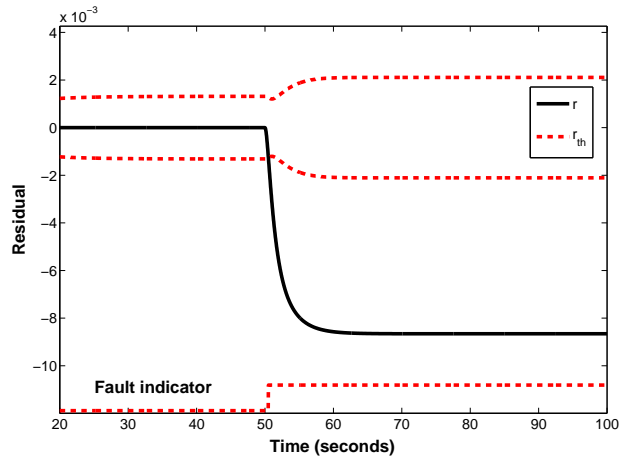

(a)

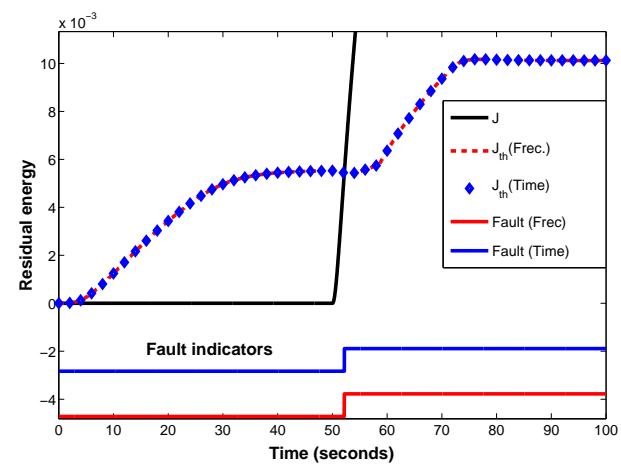

(c)

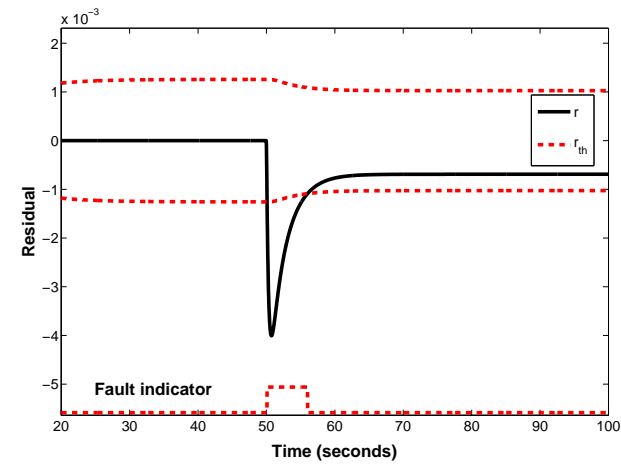

(b)

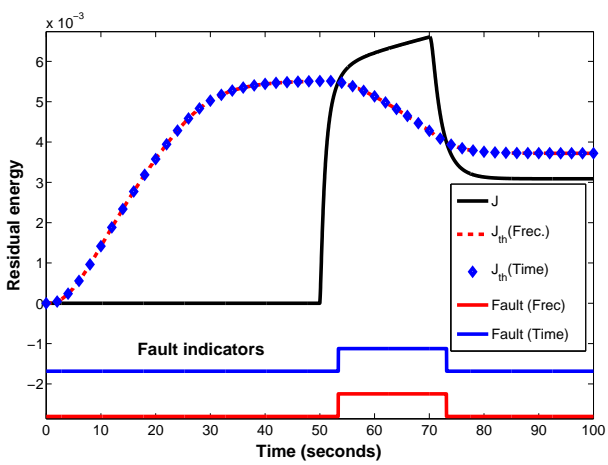

(d)

Figure 9. Fault scenario 1. Input sensor fault $f_{u_{t}}=0.12 \mathrm{~V}$ (a) Residual of azimuth angle. (b) Residual of pitch angle. (c) Residual energy of azimuth angle. (d) Residual's energy of pitch angle.

\begin{tabular}{c|c|c} 
Fault Scenario 1 & Azimuth angle & Pitch angle \\
\hline Detection Test & Det. Time & Det. Time \\
\hline \hline Basic residual & $50.50 \mathrm{~s}$ & $50.10 \mathrm{~s}$ \\
Residual energy (Freq.) & $52.18 \mathrm{~s}$ & $53.38 \mathrm{~s}$ \\
Residual energy (Time) & $52.20 \mathrm{~s}$ & $53.40 \mathrm{~s}$
\end{tabular}

Table I. Detection indexes when the input sensor fault $f_{u_{t}}=0.12 \mathrm{~V}$ is presented. 
8.4.2. Fault scenario 2 presents an additive sensor fault of the azimuth angle of beam $f_{\theta_{h}}$ :

$$
f_{\theta_{h}}(t)=\left\{\begin{array}{cl}
0 \mathrm{rad}, & \text { for } t<30 \\
0.05 \mathrm{rad}, & \text { for } t \geq 30
\end{array}\right.
$$

As in the case of the fault scenario 1, in Fig. 10(a)-(b) results from fault detection based on the basic residual evaluation (21) detect the fault after a second after the fault appearance. In Fig. 10(c)(d) results from the evaluation of the residual energy against the adaptive energy threshold $\left(J_{t h}\right)$ are presented when a window time of $T=20 \mathrm{~s}$ and frequency window of $\mathrm{W}=20 \mathrm{~Hz}$ is used. Applying the fault detection test (26), either in the time or the frequency domain, the fault is detected at $t=50.02 s$ until $t=70.01$ according to the time window used. These results are summarized for azimuth angle in the Table II.

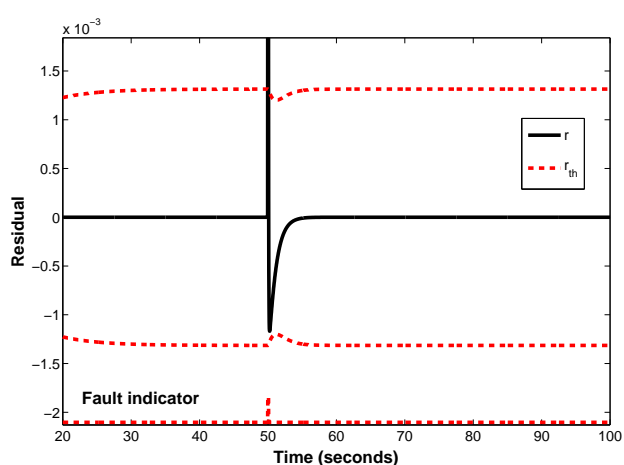

(a)

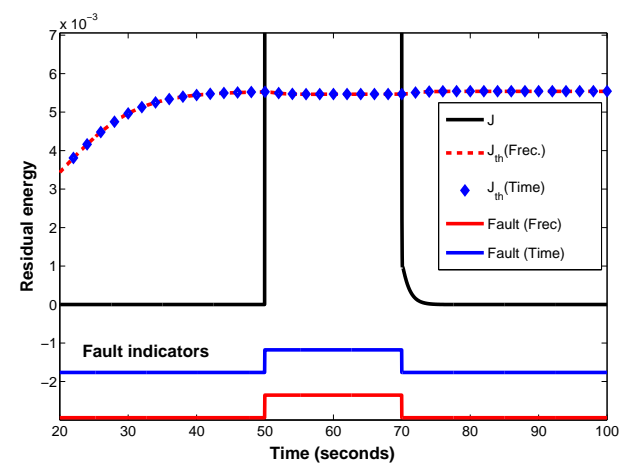

(c)

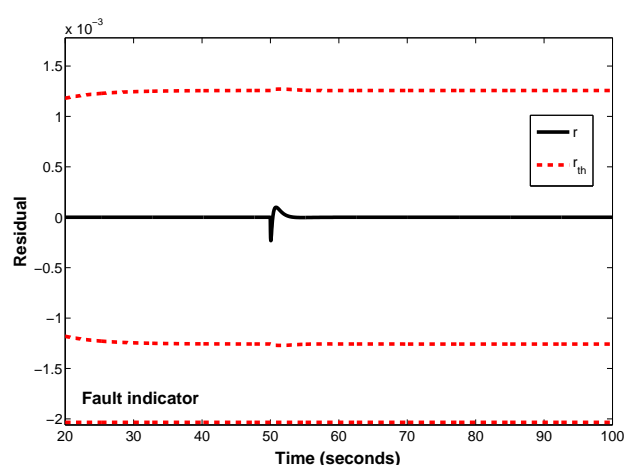

(b)

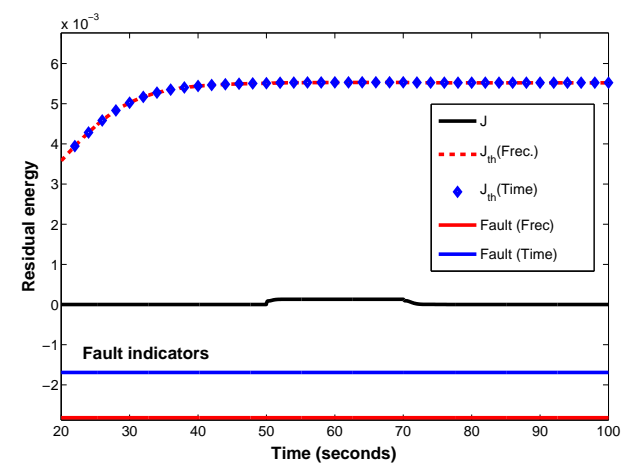

(d)

Figure 10. Output sensor fault of $f_{\theta_{h}}=0.05$ (a) Residual of azimuth angle. (b) Residual of pitch angle. (c) Residual energy of azimuth angle $(T=20 s)$. (d) Residual energy of pitch angle $(T=20 s)$.

\begin{tabular}{c|cc} 
Fault Scenario 2 & \multicolumn{2}{|c}{ Azimuth angle } \\
\hline Detection Test & Detection Instant & Detection Time \\
\hline \hline Basic residual & $50.02 \mathrm{~s}$ & $1.09 \mathrm{~s}$ \\
Residual energy (Freq.) & $50.02 \mathrm{~s}$ & $19.99 \mathrm{~s}$ \\
Residual energy (Time) & $50.02 \mathrm{~s}$ & $19.99 \mathrm{~s}$ \\
\hline
\end{tabular}

Table II. Detection indexes when the output sensor fault of $f_{\theta_{h}}=0.05 \mathrm{rad}$ is presented. 
8.4.3. Fault scenario 3 presents an additive actuator fault of the voltage of the main motor $f_{a_{m}}$ :

$$
f_{a_{m}}(t)=\left\{\begin{array}{ccc}
0, & \text { for } & t<60 \\
0.025, & \text { for } & t \geq 60
\end{array}\right.
$$

In Fig. 11(a)-(b) results from fault detection (21) based on the basic residual evaluation detect the fault at $t=60.67 \mathrm{~s}$. In Fig. 11(c)-(d) results from the evaluation of the residual energy against the adaptive energy threshold $\left(J_{t h}\right)$ are presented when a window time of $T=20 \mathrm{~s}$ and frequency window of $W=20 \mathrm{~Hz}$ is used. The fault detection test (26) in frequency domain provides equivalent results than the ones obtained in the time domain. Notice, in this fault scenario, the basic residual approach detects the fault $10 s$ before the residual energy approaches. These results are summarized for azimuth and pitch angle in the Table III.

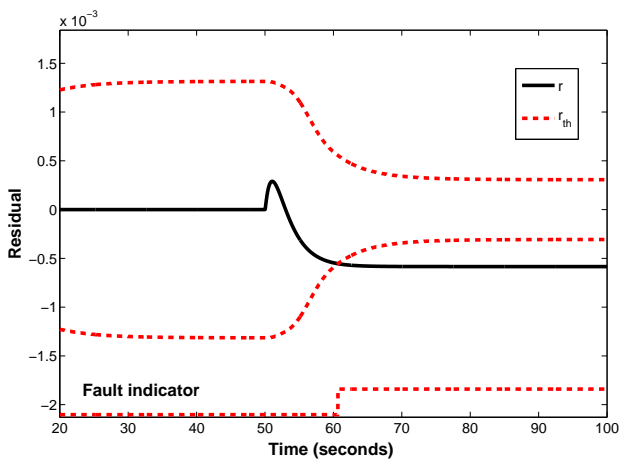

(a)

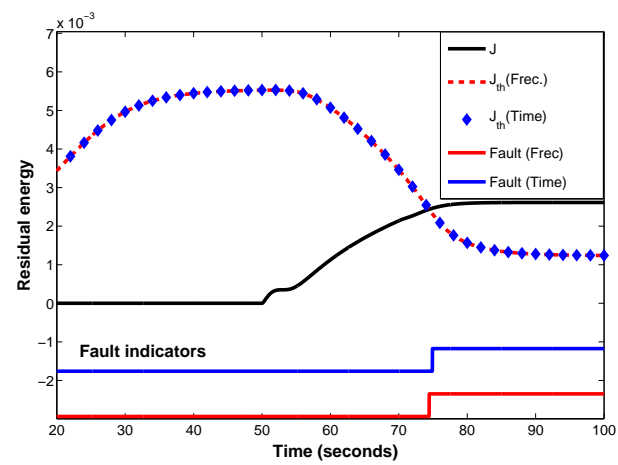

(c)

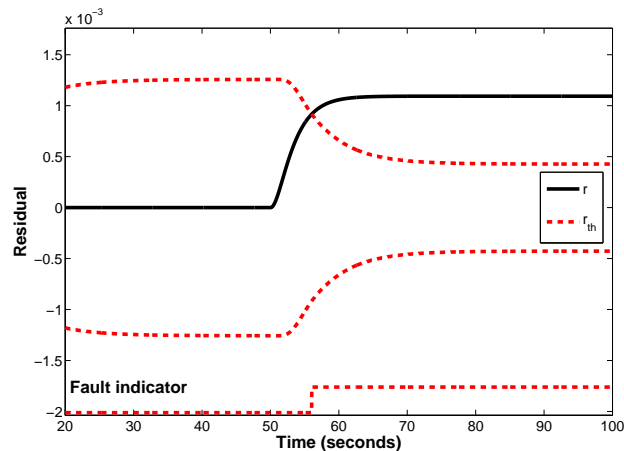

(b)

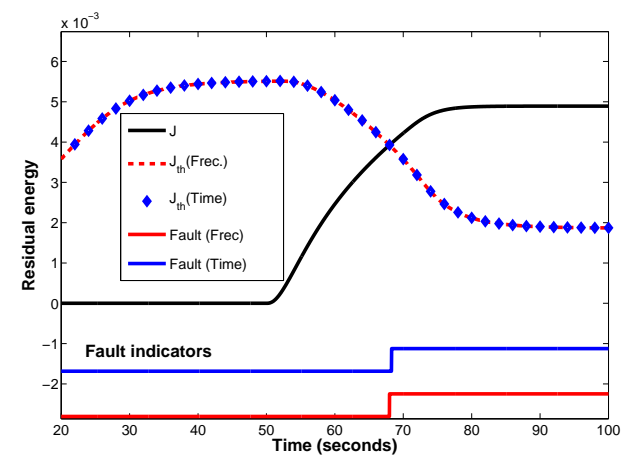

(d)

Figure 11. Fault scenario 1. Actuator fault $f_{a_{m}}=0.5$ (a) Residual of azimuth angle. (b) Residual of pitch angle. (c) Residual energy of azimuth angle $(T=15 \mathrm{~s})$. (d) Residual's energy of pitch angle $(T=15 \mathrm{~s})$.

\begin{tabular}{c|c|c} 
Fault Scenario 3 & Azimuth angle & Pitch angle \\
\hline Detection Test & Det. Instant & Det. Instant \\
\hline \hline Basic residual & $60.68 \mathrm{~s}$ & $56.05 \mathrm{~s}$ \\
Residual energy (Freq.) & $70.43 \mathrm{~s}$ & $68.00 \mathrm{~s}$ \\
Residual energy (Time) & $70.42 \mathrm{~s}$ & $68.00 \mathrm{~s}$ \\
\hline
\end{tabular}

Table III. Detection indexes when the actuator fault $f_{a_{m}}=0.025$ is presented. 


\subsection{Results discussion}

From the results obtained in the previous fault scenarios, it can be noticed that using the proposed time/frequency windows, the fault detection and energy/threshold evaluation results obtained using the time and frequency approaches proposed in this paper are equivalent from the practical point of view. These results reconfirm the ones obtained with the DAMADICS application.

\section{CONCLUSIONS}

In this paper, two approaches based on interval models for robust fault detection are proposed as means to produce energy adaptive thresholds for the residuals using techniques in time and frequency domains. The first approach proposes generating an adaptive threshold through determining the worst-case time evolution of the residual's energy using a zonotope-based algorithm. The second approach evaluates the worst-case energy evolution in the frequency domain using the Kharitonov polynomials. The paper has also related the results obtained using both approaches using the Parseval's Theorem. As result of such a comparison, it is concluded that from the theoretical point of view both approaches produce the same results when infinite time/frequency windows. In real applications, however, finite time/frequency windows should be used. The paper shows that practically equivalent results in time/frequency can be obtained when long enough time/frequency windows are used. The proposed fault detection of both approaches have been successfully applied to DAMADICS and TRMS examples allowing at the same time to prove the practical equivalence of the results obtained using the proposed approaches. As future research, the effect of noise in the performance of both approaches will be analysed.

\section{ACKNOWLEDGMENTS}

This work has been partially funded by the grant CICYT SHERECS DPI2011- 26243, by European Comission through contract i-Sense FP7-ICT-2009-6-270428 and by a grant from Consejo Nacional de Ciencia y Tecnologia (CONACyT),

\section{A. INTERVAL MODEL TIME RESPONSE USING ZONOTOPES}

In this paper, zonotopes are used to bound the set of uncertain estimated states and outputs. Let us introduce zonotopes:

Definition 3. The Minkowski sum of two sets $\mathbb{X}$ and $\mathbb{Y}$ is defined by $\mathbb{X} \oplus \mathbb{Y}=\{x+y: x \in \mathbb{X}, y \in \mathbb{Y}\}$.

Definition 4. Given a center vector $\pi \in \mathfrak{R}^{n}$ and a matrix $\mathrm{H} \in \mathfrak{R}^{n \times m}$, the Minkowski sum of the segments defined by the columns of matrix $\mathrm{H}$ is called a zonotope of order $m$. This set is represented as:

$$
\mathbb{X}=\pi \oplus H \beta^{m}=\left\{\pi+H z: z \in \beta^{m}\right\}
$$

where: $\beta^{m}$ is a unitary box, composed by $\mathrm{m}$ unitary intervals. 
Definition 5. The interval hull $\square \mathbb{X}$ of a closed set $\mathbb{X}$ is the smallest interval box that contains $\mathbb{X}$.

Given a zonotope $\mathbb{X}=\pi \oplus H \beta^{m}$, its interval hull can be easily computed by evaluating $\pi \oplus H \beta^{m}$, for all $i=1, \ldots, n$ :

$$
\square \mathbb{X}=\left\{x:\left|x_{i}-\pi_{i}\right| \leq\left\|H_{i}\right\|_{1}\right\}
$$

where $x_{i}$ and $\pi_{i}$ are $i^{\text {th }}$ components of $x$ and $\pi$, respectively, and $H_{i}$ is $i^{t h}$-row of $H$.

Interval observer estimation (33)-(34) using zonotopes can be carry out using the following result:

Theorem 1. "Zonotope Inclusion" (see [2]). Consider a family of zonotopes represented by $\mathbb{X}=\pi \oplus M \beta^{m}$ where $\pi \in \mathbb{R}^{n}$ is a real vector and $M \in \mathbb{I}^{n \times m}$ is an interval matrix. A zonotope inclusion $\diamond(\mathbb{X})$ is defined by: $\diamond(\mathbb{X})=\pi \oplus(\operatorname{mid}(M) G)\left(\begin{array}{c}\beta^{m} \\ \beta^{n}\end{array}\right)=\pi \oplus J \beta^{n+m}$

where $G \in \mathfrak{R}^{n \times n}$ is a diagonal matrix that satisfies: $G_{i i}=\sum_{j=1}^{m} \frac{\operatorname{diam}\left(M_{i j}\right)}{2}, i=1,2, \ldots, n$, mid denotes the center and diam the diameter of the interval according to [39]. Under this definition, $\mathbb{X} \subseteq \diamond(\mathbb{X})$.

Note that this result is the generalization of the linear image of a zonotope given by the following property:

Property 1. "Zonotope Linear Image Transformation" (see [15]) Consider a zonotope represented by $\mathbb{X}=\pi \oplus H \beta^{m}$ where $\pi \in \mathfrak{R}^{n}$ is a vector and $H \in \mathfrak{R}^{n \times m}$ is a matrix. The image of the zonotope $\mathbb{X}$ through a linear transformation $T \in \mathfrak{R}^{n \times n}$ is a zonotope $\mathbb{Y}$ defined by:

$$
\mathbb{Y}=q \oplus N \beta^{m}
$$

where: $q=T \pi$ and $N=T H$. The existence of this property motivates the use of zonotopes to propagate the uncertainty in dynamic systems.

Using Theorem 1 , the zonotope $\mathbb{X}_{k+1}^{e}$ that bounds the trajectory of the system at instant $k+1$, is computed from the previous approximating zonotope at time instant $k, \mathbb{X}_{k}^{e}=\pi(k) \oplus H_{k} \beta^{m}$, using the natural interval extension of (34) as suggested by [39] and the zonotope inclusion operator, as a generalization of Kühn's method (see [32]):

$$
\mathbb{X}_{k+1}^{e}=\pi(k+1) \oplus H_{k+1} \beta^{r}
$$

where:

$$
\begin{aligned}
\pi(k+1) & =\operatorname{mid}\left(A_{0}(\theta)\right) \pi(k)+\operatorname{mid}\left(B_{0}(\theta)\right) v(k) \\
H_{k+1} & =\left(J_{1} \quad J_{2} \quad J_{3}\right) \\
J_{1} & =\operatorname{seg}\left(\diamond\left(A_{0}(\theta) H_{k}\right)\right) \\
J_{2} & =\frac{\operatorname{diam}\left(A_{o}(\theta)\right)}{2} \pi(k) \\
J_{3} & =\frac{\operatorname{diam}\left(B_{o}(\theta)\right)}{2} v(k)
\end{aligned}
$$

$J_{1}$ is calculated using the zonotope inclusion operator and $\operatorname{seg}(\mathbb{X})=M$ considering that $\mathbb{X}=\pi \oplus$ $M \beta^{m_{z}}$ is a zonotope (see Definition 4).

It is important to notice that the set of estimated states has an increasing number of segments generating the zonotope $\mathbb{X}_{k+1}^{e}$ using this method. In order to control the domain complexity, a 
reduction step is thus implemented. Here we use the method proposed in [14] to reduce the zonotope complexity.

Analogously, Theorem 1 can be used to obtain the set $\mathbb{Y}_{k}^{e}$ from the set $\mathbb{X}_{k}^{e}$ through the expression for the estimated output (34).

\section{B. INTERVAL MODEL FREQUENCY RESPONSE USING KHARITONOV POLYNOMIALS}

Let

$$
G(j \omega, \theta)=\frac{P_{1}(j \omega, \theta)}{P_{2}(j \omega, \theta)}
$$

be a transfer function for which maximum and minimum values for module and angle have to be determined with respect to a set of uncertain parameters $\theta^{\mathbb{I}}$. Then:

$$
\max (|G(j \omega)|)=\frac{\max \left(\left|P_{1}(j \omega)\right|\right)}{\min \left(\left|P_{2}(j \omega)\right|\right)}, \quad \min (|G(j \omega)|)=\frac{\min \left(\left|P_{1}(j \omega)\right|\right)}{\max \left(\left|P_{2}(j \omega)\right|\right)}
$$

and

$$
\begin{aligned}
\max (\angle G(j \omega)) & =\max \left(\angle P_{1}(j \omega)\right)-\min \left(\angle P_{2}(j \omega)\right), \\
\min (\angle G(j \omega)) & =\min \left(\angle P_{1}(j \omega)\right)-\max \left(\angle P_{2}(j \omega)\right) .
\end{aligned}
$$

It is clear that it is necessary to know how to determine the maximum and the minimum values for the module and the angle of a general interval polynomial $P(j \omega)$. The previous results to Kharitonov's Theorem give some rules or conditions needed to this representation. At a given frequency it is correct to write that all the possible images of the interval polynomial

$$
P\left(j \omega_{k}\right)=\left[p_{q}^{-}, p_{q}^{+}\right]\left(j \omega_{k}\right)^{q}+\left[p_{q-1}^{-}, p_{q-1}^{+}\right]\left(j \omega_{k}\right)^{q-1}+\cdots+\left[p_{q}^{-}, p_{q}^{+}\right]\left(j \omega_{k}\right)+\left[p_{0}^{-}, p_{0}^{+}\right]
$$

are contained in a rectangle in the complex plane whose vertices are the four Kharitonov polynomials:

$$
\begin{aligned}
& P_{1}\left(j \omega_{k}\right)=p_{0}^{+}+p_{1}^{+}\left(j \omega_{k}\right)+p_{2}^{-}\left(j \omega_{k}\right)^{2}+p_{3}^{-}\left(j \omega_{k}\right)^{3}+p_{4}^{+}\left(j \omega_{k}\right)^{4}+\cdots, \\
& P_{2}\left(j \omega_{k}\right)=p_{0}^{-}+p_{1}^{-}\left(j \omega_{k}\right)+p_{2}^{+}\left(j \omega_{k}\right)^{2}+p_{3}^{+}\left(j \omega_{k}\right)^{3}+p_{4}^{-}\left(j \omega_{k}\right)^{4}+\cdots, \\
& P_{3}\left(j \omega_{k}\right)=p_{0}^{-}+p_{1}^{+}\left(j \omega_{k}\right)+p_{2}^{+}\left(j \omega_{k}\right)^{2}+p_{3}^{-}\left(j \omega_{k}\right)^{3}+p_{4}^{-}\left(j \omega_{k}\right)^{4}+\cdots, \\
& P_{4}\left(j \omega_{k}\right)=p_{0}^{+}+p_{1}^{-}\left(j \omega_{k}\right)+p_{2}^{-}\left(j \omega_{k}\right)^{2}+p_{3}^{+}\left(j \omega_{k}\right)^{3}+p_{4}^{+}\left(j \omega_{k}\right)^{4}+\cdots,
\end{aligned}
$$

If these vertices are plotted in complex plane $\mathbb{C}$ it will result something like this:

Note that there are more than nine possible relative situations between the Kharitonov rectangle and the axis of the complex plane, nine of them are illustrated in Figure 13.

We say "more than" nine situations because when a rectangle intersects with at least one axis it can do so either in a symmetric or asymmetric way. According to this consideration, the set of rules is presented in Algorithm 1.

$\overline{\mathbb{T}}$ For simplicity in the notation, the dependency of all transfer functions and polynomials will be omitted in the sequel 


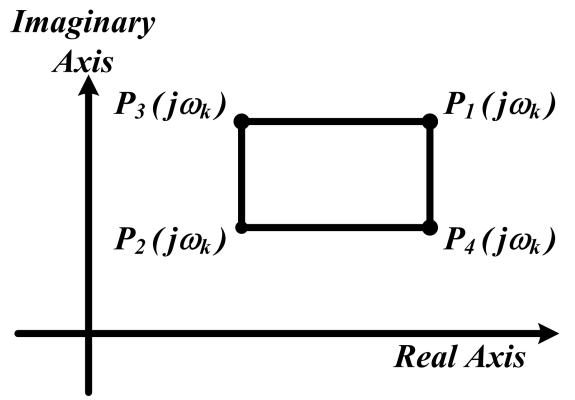

Figure 12. Example of a plot of $P\left(j \omega_{k}\right)$ in $\mathbb{C}$

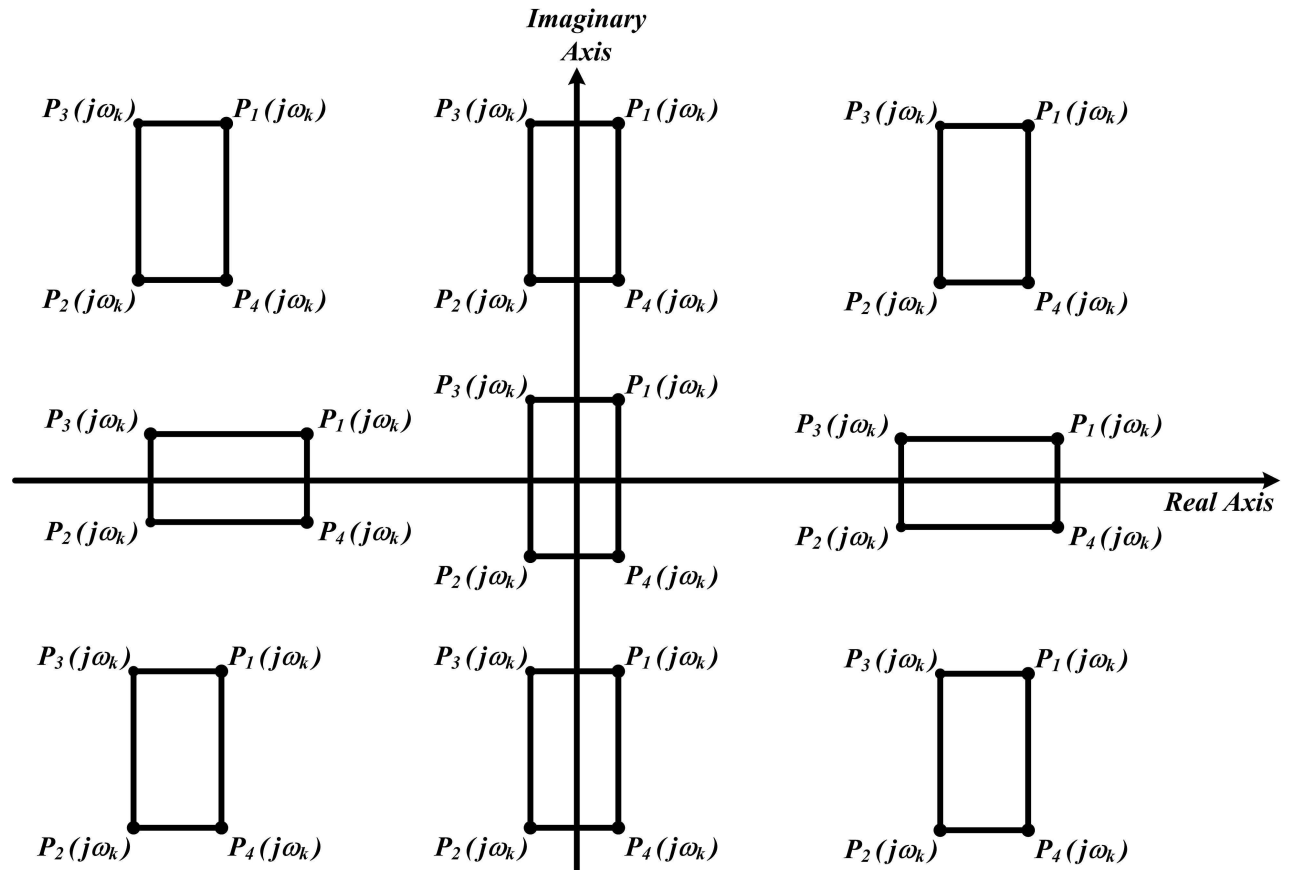

Figure 13. Possible relative situations between a plot of $P\left(j \omega_{k}\right)$ and axis in $\mathbb{C}$

Algorithm 2. Set of the rules

1: if $\operatorname{Re}\left\{K_{2}\left(j \omega_{k}\right)\right\}>0$ and $\operatorname{Im}\left\{K_{2}\left(j \omega_{k}\right)\right\}>0$

2: $\quad\left|P\left(j \omega_{k}\right)\right|^{+}=\left|K_{1}\left(j \omega_{k}\right)\right|$

3: $\quad\left|P\left(j \omega_{k}\right)\right|^{-}=\left|K_{2}\left(j \omega_{k}\right)\right|$

4: $\quad \angle P\left(j \omega_{k}\right)^{+}=\angle K_{3}\left(j \omega_{k}\right)$

5: $\quad \angle P\left(j \omega_{k}\right)^{-}=\angle K_{4}\left(j \omega_{k}\right)$

6: else if $\operatorname{Re}\left\{K_{2}\left(j \omega_{k}\right)\right\} \leq 0$ and $\operatorname{Im}\left\{K_{2}\left(j \omega_{k}\right)\right\}>0$ and $\operatorname{Re}\left\{K_{4}\left(j \omega_{k}\right)\right\} \geq 0$

7: $\quad\left|P\left(j \omega_{k}\right)\right|^{-}=\left|K_{2}\left(j \omega_{k}\right)\right|$

8: $\quad \angle P\left(j \omega_{k}\right)^{+}=\angle K_{2}\left(j \omega_{k}\right)$

9: $\quad \angle P\left(j \omega_{k}\right)^{-}=\angle K_{4}\left(j \omega_{k}\right)$

10: $\quad$ if $\operatorname{Re}\left\{K_{2}\left(j \omega_{k}\right)\right\}+\operatorname{Re}\left\{K_{4}\left(j \omega_{k}\right)\right\} \geq 0$

11: $\quad\left|P\left(j \omega_{k}\right)\right|^{+}=\left\|K_{1}\left(j \omega_{k}\right)\right\|$ 
12: else

13: $\quad\left|P\left(j \omega_{k}\right)\right|^{+}=\left\|K_{3}\left(j \omega_{k}\right)\right\|$

14: end if

15: else if $\operatorname{Re}\left\{K_{4}\left(j \omega_{k}\right)\right\}<0$ and $\operatorname{Im}\left\{K_{4}\left(j \omega_{k}\right)\right\}>0$

16: $\quad\left|P\left(j \omega_{k}\right)\right|^{+}=\left|K_{3}\left(j \omega_{k}\right)\right|$

17: $\quad\left|P\left(j \omega_{k}\right)\right|^{-}=\left|K_{4}\left(j \omega_{k}\right)\right|$

18: $\quad \angle P\left(j \omega_{k}\right)^{+}=\angle K_{2}\left(j \omega_{k}\right)$

19: $\quad \angle P\left(j \omega_{k}\right)^{-}=\angle K_{4}\left(j \omega_{k}\right)$

20: else if $\operatorname{Re}\left\{K_{1}\left(j \omega_{k}\right)\right\}<0$ and $\operatorname{Im}\left\{K_{2}\left(j \omega_{k}\right)\right\} \leq 0$ and $\operatorname{Im}\left\{K_{3}\left(j \omega_{k}\right)\right\} \geq 0$

21: $\quad\left|P\left(j \omega_{k}\right)\right|^{-}=\left|K_{1}\left(j \omega_{k}\right)\right|$

22: $\quad \angle P\left(j \omega_{k}\right)^{+}=\angle K_{4}\left(j \omega_{k}\right)$

23: $\quad \angle P\left(j \omega_{k}\right)^{-}=\angle K_{1}\left(j \omega_{k}\right)$

24: $\quad$ if $\operatorname{Im}\left\{K_{2}\left(j \omega_{k}\right)\right\}+\operatorname{Im}\left\{K_{3}\left(j \omega_{k}\right)\right\} \geq 0$

25: $\quad\left|P\left(j \omega_{k}\right)\right|^{+}=\left|K_{3}\left(j \omega_{k}\right)\right|$

26: else

27: $\quad\left|P\left(j \omega_{k}\right)\right|^{+}=\left|K_{2}\left(j \omega_{k}\right)\right|$

28: end if

29: else if $\operatorname{Re}\left\{K_{1}\left(j \omega_{k}\right)\right\}<0$ and $\operatorname{Im}\left\{K_{1}\left(j \omega_{k}\right)\right\}<0$

30: $\quad\left|P\left(j \omega_{k}\right)\right|^{+}=\left|K_{2}\left(j \omega_{k}\right)\right|$

31: $\quad\left|P\left(j \omega_{k}\right)\right|^{-}=\left|K_{1}\left(j \omega_{k}\right)\right|$

32: $\quad \angle P\left(j \omega_{k}\right)^{+}=\angle K_{4}\left(j \omega_{k}\right)$

33: $\quad \angle P\left(j \omega_{k}\right)^{-}=\angle K_{3}\left(j \omega_{k}\right)$

34: else if $\operatorname{Re}\left\{K_{3}\left(j \omega_{k}\right)\right\} \leq 0$ and $\operatorname{Im}\left\{K_{3}\left(j \omega_{k}\right)\right\}<0$ and $\operatorname{Im}\left\{K_{1}\left(j \omega_{k}\right)\right\} \geq 0$

35: $\quad\left|P\left(j \omega_{k}\right)\right|^{-}=\left|K_{3}\left(j \omega_{k}\right)\right|$

36: $\quad \angle P\left(j \omega_{k}\right)^{+}=\angle K_{1}\left(j \omega_{k}\right)$

37: $\quad \angle P\left(j \omega_{k}\right)^{-}=\angle K_{3}\left(j \omega_{k}\right)$

38: $\quad$ if $\operatorname{Re}\left\{K_{2}\left(j \omega_{k}\right)\right\}+\operatorname{Re}\left\{K_{4}\left(j \omega_{k}\right)\right\} \geq 0$

39: $\quad\left|P\left(j \omega_{k}\right)\right|^{+}=\left|K_{4}\left(j \omega_{k}\right)\right|$

40: else

41: $\quad\left|P\left(j \omega_{k}\right)\right|^{+}=\left|K_{2}\left(j \omega_{k}\right)\right|$

42: end if

43: else if $\operatorname{Re}\left\{K_{3}\left(j \omega_{k}\right)\right\}>0$ and $\operatorname{Im}\left\{K_{3}\left(j \omega_{k}\right)\right\}<0$

44: $\quad\left|P\left(j \omega_{k}\right)\right|^{+}=\left|K_{2}\left(j \omega_{k}\right)\right|$

45: $\quad\left|P\left(j \omega_{k}\right)\right|^{-}=\left|K_{1}\left(j \omega_{k}\right)\right|$

46: $\quad \angle P\left(j \omega_{k}\right)^{+}=\angle K_{4}\left(j \omega_{k}\right)$

47: $\quad \angle P\left(j \omega_{k}\right)^{-}=\angle K_{3}\left(j \omega_{k}\right)$

48: else if $\operatorname{Re}\left\{K_{2}\left(j \omega_{k}\right)\right\}>0$ and $\operatorname{Im}\left\{K_{2}\left(j \omega_{k}\right)\right\} \leq 0$ and $\operatorname{Im}\left\{K_{3}\left(j \omega_{k}\right)\right\} \geq 0$

49: $\quad\left|P\left(j \omega_{k}\right)\right|^{-}=\left|K_{2}\left(j \omega_{k}\right)\right|$

50: $\quad \angle P\left(j \omega_{k}\right)^{+}=\angle K_{4}\left(j \omega_{k}\right)$

51: $\quad \angle P\left(j \omega_{k}\right)^{-}=\angle K_{1}\left(j \omega_{k}\right)$

52: $\quad$ if $\operatorname{Im}\left\{K_{2}\left(j \omega_{k}\right)\right\}+\operatorname{Im}\left\{K_{3}\left(j \omega_{k}\right)\right\} \geq 0$

53: $\quad\left|P\left(j \omega_{k}\right)\right|^{+}=\left|K_{1}\left(j \omega_{k}\right)\right|$

54: else 


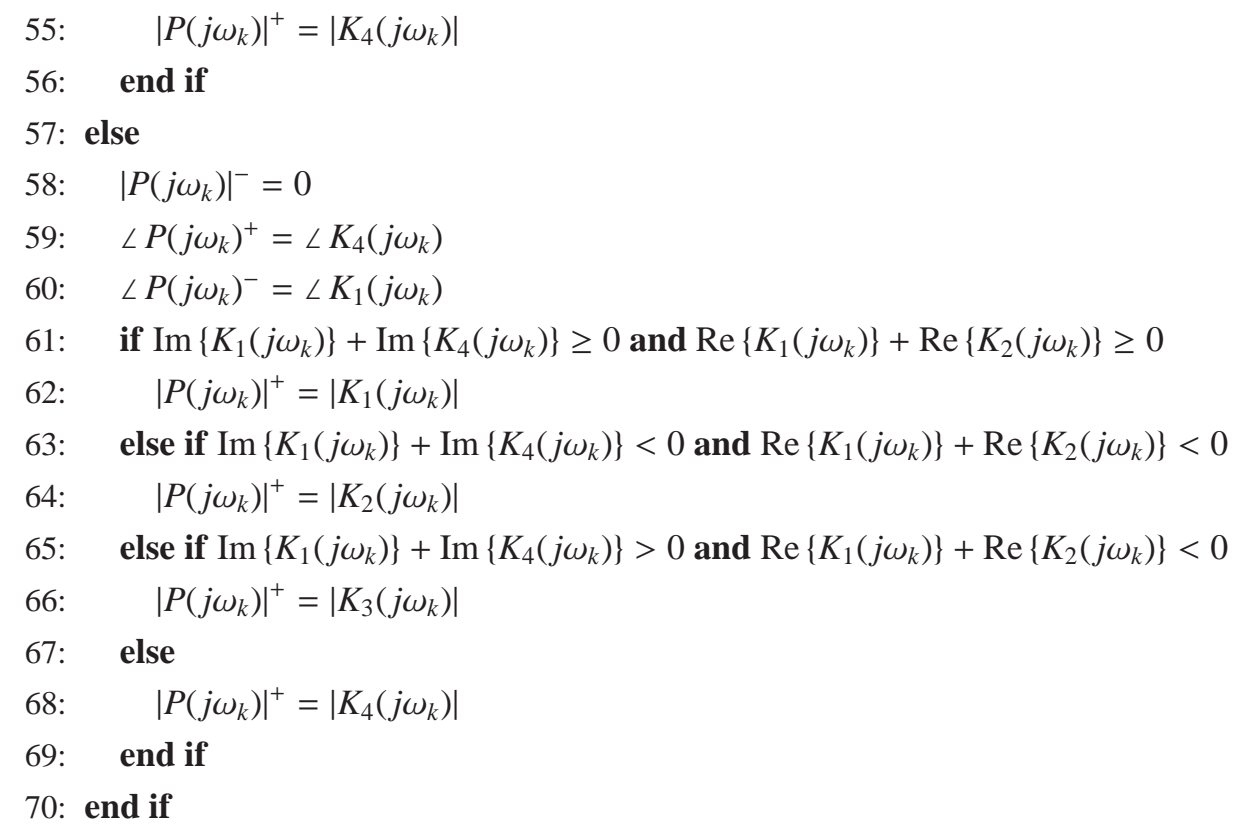

Once it is known how to determine the maximum and the minimum value of the module and angle of a given interval polynomial, it will be easy to determine the maximum and minimum module and angle of an interval transfer function. Consequently, the set of inclusion rules as constrains for the optimization problem can now be written.

References

1. O. Adrot, D. Maquin, and J. Ragot. Bounding Approaches to Fault Detection of Uncertain Dynamic Systems. In IFAC SAFEPROCESS'O0, Hungary, 2000.

2. T. Alamo, J.M. Bravo, and E.F. Camacho. Guaranteed State Estimation by Zonotopes. Automatica, 41(6):1035$1043,2005$.

3. A. Ambardar. Analog and Digital Signal Processing. International Thomson Publishing Company, 1995.

4. P. Apkarian, P. Gahinet, and G. Becker. Self-scheduled $H_{\infty}$ Control of Linear Parameter-Varying Systems: A Design Example. Automatica, 31(9):1251 - 1261, 1995.

5. J. Armengol, J. Vehi, L. Trave-Massuyes, and M. A. Sainz. Application of Modal Intervals to the Generation of Error-bounded Envelopes. Reliable Computing, 7(2):171-185, 2001.

6. B.R. Barsmish and J. Sankaran. The Propagation of Uncertainty via Politopes. IEEE Transactions on Automatic Control, 24(2), 1979.

7. M. Bartys. Specification of Actuators Intended to use for Benchmark Definition, v. 1.02., 2002.

8. M. Bartys, R.J. Patton, M. Syfert, S. de las Heras, and J. Quevedo. Introduction to the DAMADICS actuator FDI Benchmark Study. Control Engineering Practice, 14(6):577-596, 2006.

9. M. Berz and K. Makino. Verified Integration of ODEs and Flows using Differential Algebraic Methods on Highorder Taylor Models. Reliable Computing, 4:361-369, 1998.

10. A. Bonarini and G. Bontempi. A Qualitative Simulation Approach to Fuzzy Dynamical Models. ACM Transactions on Modelling and Computer Simulation (TOMACS), 4(4):258-313, 1994.

11. J. Chen and R. J. Patton. Robust Model-based Fault Diagnosis for Dynamic Systems. Kluwer Academic Publishers, 1999.

12. M. Chilali and P. Gahinet. $H_{\infty}$ Design with Pole Placement Constraints: An LMI Approach. IEEE Transactions on Automatic Control, 41(3):358-367, 1996.

13. M. Chilali, P. Gahinet, and P. Apkarian. Robust Pole Placement in LMI Regions. IEEE Transactions on Automatic Control, 44(12):2257-2270, December 1999. 
14. C. Combastel. A State Bounding Observer based on Zonotopes. In European Control Conference, 2003.

15. C. Combastel. A State Bounding Observer for Uncertain Non-linear Continuous-time Systems based on Zonotopes. 44th IEEE Conference on Decision and Control and European Control Conference. CDC-ECC'05., 2005.

16. Y. Deville, M. Jansen, and Van Hentenryck. Consistency Techniques in Ordinary Differential Equations. Maher and Puget eds: Principles and Practice of Constraint Programming (CP98), 4:162-176, 1998.

17. S.X. Ding. Model-based Fault Diagnosis Techniques: Design Schemes, Algorithms, and Tools. Springer, 2008.

18. X. Ding and P.M. Frank. Frequency Domain Approach and Threshold Selector for Robust Model-based Fault Detection and Isolation. In Proceedings of IFAC/IMACS SafeProcess'91, pages 307-312, Baden-Baden, Germany, 1991.

19. X. Ding and L. Guo. On Observer based Fault-Detection. In Proceedings of IFAC SafeProcess'97, pages 112-120, Hull, UK, 1997.

20. L. El Ghaoui and G. Calafiore. Worst-Case Simulation of Uncertain Systems. Lecture Notes in Control and Information Sciences, 245:134-146, 1999.

21. A. Emami-Naeini, M. M. Akhter, and S. M. Rock. Effect of Model Uncertainty of Failure Detection: The Threshold Selector. IEEE Transactions on Automatic Control, 33(12):1106-1115, 1988.

22. Feedback Instruments Ltd, Crowborough, UK. Twin Rotor MIMO System Advanced Teaching Manual 1 (33-0074M5), 1998

23. N. Femia and G. Spagnuolo. True Worst-case Circuit Tolerance Analysis using Genetic Algorithms and Affine Arithmetic. IEEE Transactions on Circuits and Systems I: Fundamental Theory and Applications, 47(9):12851296, 2000.

24. P. M. Frank and X. Ding. Optimally Robust Residual Generation and Evaluation for Model-based Fault Diagnosis. Automatica, 30(5):789-804, 1994.

25. J. Gertler. Fault Detection and Diagnosis in Engineering Systems. Marcel Dekker, New York, 1998.

26. J. L. Gouzé, A. Rapaport, and M. Z. Hadj-Sadok. Interval Observers for Uncertain Biological Systems. Journal of Ecological Modelling, 133:45-56, 2000.

27. F. Hamelin and D. Sauter. Robust Fault Detection in Uncertain Dynamic Systems. Automatica, 36(11):1747-1754, 2000.

28. D. T. Horak. Failure Detection in Dynamic Systems with Modelling Errors. Journal of Guidance, Control, and Dynamics, 11(6):508-516, 1988.

29. A. Johansson, M. Bask, and T. Norlander. Dynamic threshold generators for robust fault detection in linear systems with parameter uncertainty. Automatica, 42:1095 - 1106, 2006.

30. W. Kühn. Rigorously Computed Orbits of Dynamical Systems without the Wrapping Effect. Computing, 61:4767, 1988.

31. L. V. Kolev. Interval Methods for Circuit Analysis. Singapore: World Scientific, 1993.

32. W. Kuhn. Rigorously Computed Orbits of Dynamical Systems without the Wrapping Effect. Computing, 61(1):4767, 1998.

33. B. Kuipers. Qualitative Reasoning - Modelling and Simulation with Incomplete Knowledge. MIT Press. Cambridge, MA, 1994.

34. A. Levkovich, E. Zeheb, and N. Cohen. Frequency Response Envelopes of a Family of Uncertain Continuous-Time Systems. IEEE Transactions on Circuits and Systems I: Fundamental Theory and Applications, 42:156-165, 1995.

35. R. J. Lohner. Enclosing the Solution of Ordinary Initial and Boundary Value Problems. Computerarithmetic: Scientific Computation and Programming Languages, pages 255-286, 1987.

36. D. Maksarov and J.P. Norton. State Bounding with Ellipsoidal Set Description of the Uncertainty. International Journal of Control, 65 (5):847 - 866, 1996.

37. J. Meseguer, V. Puig, and T. Escobet. Observer Gain Effect in Linear Interval Observer-based Fault Detection. 6th IFAC Symposium on Fault Detection, Supervision and Safety of Technical Processes (SAFEPROCESS), 6, 2006.

38. M. Milanese, J. Norton, H. Piet-Lahanier, and E. Walter, editors. Bounding Approaches to System Identification. Springer, 1996.

39. R.E. Moore. Interval Analysis. Prentice Hall, 1966.

40. R.E. Moore. Methods and Applications of Interval Analysis. SIAM, Philadelphia, 1979.

41. N. S. Nedialkov, K. R. Jackson, and G. F. Corliss. Validated Solutions of Initial Value Problems for Ordinary Differential Equations. Applied Mathematics and Computation, 105:21-68, 1999.

42. A. Neumaier. The Wrapping Effect, Ellipsoid Arithmetic, Stability and Confidence Regions. Computing Supplementum, 9:175-190, 1993.

43. S. Ploix, O. Adrot, and J. Ragot. Parameter Uncertainty Computation in Static Linear Models. Proceedings of the 38th IEEE Conference on Decision and Control, 2:1916-1921, 1999. 
44. V. Puig, J. Quevedo, and T. Escobet. Robust Fault Detection Approaches using Interval Models. In IFAC World Congress (b’02), Barcelona, Spain, 2002.

45. V. Puig, J. Saludes, and J. Quevedo. A New Algorithm for Adaptive Threshold Generation in Robust Fault Detection Based on a Sliding Window and Global Optimization. In Proceedings of European Control Conference 1999, ECC'99, September 1999.

46. V. Puig, J. Saludes, and J. Quevedo. Worst-Case Simulation of Discrete Linear Time-Invariant Interval Dynamic Systems. Reliable Computing, 9, 2003.

47. F. Rambeaux, F. Hamelin, and D. Sauter. Optimal Thresholding for Robust Fault Detection of Uncertain Systems. International Journal of Robust and Nonlinear Control, 10:1155-1173, 2000.

48. M. Rodrigues, D. Theilliol, and D. Sauter. Design of an Active Fault Tolerant Control and Polytopic Unknown Input Observer for Systems described by a Multi-Model Representation. 44th IEEE Conference on Decision and Control and European Control Conference ECC, Sevilla, Spain, 2005.

49. B. Tibken and E. P. Hofer. Simulation of Controlled Uncertain Nonlinear Systems. Applied Mathematics and Computation, 70:329-338, September 1995.

50. X. Zhang, M. Polycarpou, and T. Parisini. Fault diagnosis of a class of uncertain time delay systems with Lipschitz nonlinearities. Automatica, 46:290-299, 2010. 